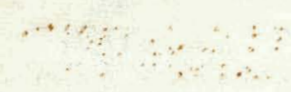

MAGNETIC EXCITATIONS ABOVE THE CRITICAL TEMPERATURE

\author{
Paul Arthur Swanson
}

Ph. D. Thesis Submitted to Iowa State University

\author{
Ames Laboratory, ERDA \\ Iowa State University \\ Ames, Iowa 50011
}

Date Transmitted: October 1975

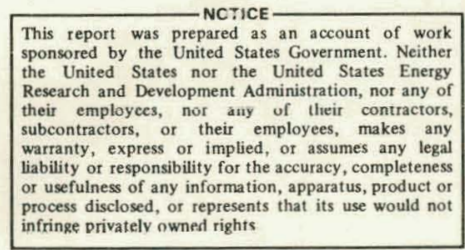

PREPARED FOR THE U.S. ENERGY RESEARCH AND DEVELOPMENT ADMINISTRATION UNDER CONTRACT NO. W-7405-eng-82

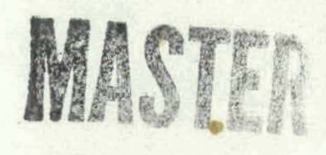




\section{DISCLAIMER}

This report was prepared as an account of work sponsored by an agency of the United States Government. Neither the United States Government nor any agency Thereof, nor any of their employees, makes any warranty, express or implied, or assumes any legal liability or responsibility for the accuracy, completeness, or usefulness of any information, apparatus, product, or process disclosed, or represents that its use would not infringe privately owned rights. Reference herein to any specific commercial product, process, or service by trade name, trademark, manufacturer, or otherwise does not necessarily constitute or imply its endorsement, recommendation, or favoring by the United States Government or any agency thereof. The views and opinions of authors expressed herein do not necessarily state or reflect those of the United States Government or any agency thereof. 


\section{DISCLAIMER}

Portions of this document may be illegible in electronic image products. Images are produced from the best available original document. 
This report was prepared as an account of work sponsored by the United States Government. Neither the United States nor the United States Energy Research and Development Administration, nor any of their employees, nor any of their contractors, subcontractors, or their employees, makes any warranty, express or implied, or assumes any legal liability or responsibility for the accuracy, completeness, or usefulness of any information, apparatus, product or process disclosed, or represents that its use would not inf ringe privately. owned rights.

Available from: National Technical Information Service U. S. Department of Commerce P.O. Box 1553

Springfield, VA 22161

Price: Microfiche $\$ 2.25$ 
TABLE OF CONTENTS

Page

Abstract

iv.

1. INTRODUCTION

1

12

11. REVIEW OF PREVIOUS THEORIES :

IV. CALCULATION OF THE LOWEST ORDER COEFFICIENTS

IN THE SERIES EXPANSION FOR THE TRANSVERSE AND

LONGITUDINAL CORRELATION FUNCTIONS

48

A. Importance of Short Range Order above the Critical Temperature

48

B. Determination of $\mathrm{G}^{\top}(\overrightarrow{\mathrm{q}}, w) \quad \ldots 5$

c. Determination of $\mathrm{G}^{Z Z}(\vec{q}, \omega) \quad \therefore 67$

D. Transverse and Longitudinal Contributions to
the Line Shape

E. Comparison with Previous Theories. 72

v. DISCUSSION OF RESULTS 77

vI. CONCLUSION 83

VII. REFERENCES 86

7.II. ACKNOWLEDGMENTS . . . . 89

1X. APPENDIX A: CALCULATION OF $\left\langle G_{\rightarrow}^{(0)}\right\rangle^{+-}$AND $\left\langle G_{\rightarrow}^{(3)}\right\rangle^{+-} 90$

$X$. APPENDIX B: TEMPERATURE DEPENDENCE OF THE BACKGROUND TERMS IN $\langle\underset{\vec{q}}{(U)}\rangle^{+-}$AND $\underset{\vec{q}}{\left\langle G^{(2)}\right\rangle^{+-}}$

XI. APPENDIX C: CALCULATION OF $\left.\underset{\vec{q}}{\left\langle G^{(0)}\right.}\right\rangle^{Z Z}$ AND $\left.\underset{\vec{q}}{\left\langle G^{(2)}\right.}\right\rangle^{Z Z}$ 


\title{
Magnetic excitations above the \\ critical temperature
}

\author{
Paul Arthur Swanson \\ Under the supervision of Samuel H. Liu \\ From the Department of Physics \\ lowa State University
}

In this work, a new approach has been developed to obtain an approximate expression for the dynamical spin correlation function. This involves the expansion of the dynamical correlation function in a Taylor series in powers of the time whose coefficients are equal to progressively higher order static multiple spin correlation functions. The lowest order coefficients in this series expansion are then evaluated through the use of a decoupling scheme which explicitly takes into account positional spin correlations above the critical temperature. As a consequence of this method, whien $r<q^{-1}<\lambda$ where $r$ is the range of the exchange interaction, $\lambda$ is the two-spin correlation length, and $\vec{q}$ is the wave vector of the spin fluctuations, the dominant contribution to these coefficients is found to be caused by spin clusters involving strongly correlated spins. The degree of local order in this spin system is measured by a time-independent local order parameter ${ }^{\prime} p_{i j}$ ' the static two-spin correlation function. The resulting expressions for the zeorth first, and second order coefficients are incorporated as leading terms in a resummation that is used to obtain an approximate

For a discussion of local order in one dimensional magnetic systems see F. B. McLean and M. Blume, Phys. Rev. B7, 1149 (1973). 



\section{INTRODUCTION}

In examining the low temperature thermodynamic behavior of ferromagnets $B l o c h{ }^{1,2}$ found that the eigenfunctions corresponding to the excitations from the ground state of the exchange Hamiltonian were not characterized by states involving one spin reversal but rather by a linear combination of these states each containing a spin reversal located at a different magnetic ion site. The wave nature of these proper linear combinations was demonstrated by slater $^{3}$ in showing that the quantum mechanical problem of one spin reversal could be exactly solved. Thus physically a spin wave may be viewed as a spin reversal propagating through the crystal via exchange interactions between neighboring spins. In Bloch's calculation it was assumed that the number of spin waves excited at low temperatures was so small that all interactions between them could be neglected, and consequently the low-lying excited states in a ferromagnet could be adequately approximated by a linear superposition of these sinusoidal spin waves.

Early attempts 4,5 at studying deviations from Bloch's theory due to spin wave interactions seemed to indicate that the region of validity for spin wave theory was closely confined to temperatures near absolute zero $\left(\sim 0.1 T_{c}\right)$. This result was obtained by Van Kranendonk ${ }^{6}$ who analyzed spin wave interactions within the framewark of a gas of spin deviations Interacting via a hard core potential. Dyson, 7,8 on the other hand, in studying the interaction between two spin waves not only found that the linear superposition principle discussed by Bloch was not quite correct but also that Van Kranendonk had over-estimated the interaction 
between spin waves. He distinguished two types of these interactions. The first is a consequence of the nonorthogonality of the spin wave states containing more than one spin wave and is called the kinematical interaction. This is a repulsive interaction whose physical significance is that no more than $2 S$ units of spin can be attached to the same ion simultaneously. The other interaction arises from the fact that the exchange Hamiltonian is not diagonal in these states. This is called the dynamical interaction which causes a shift in the spin wave energy due to the presence of other spin waves. Dyson has shown that the kinematical interaction makes no contribution to the temperature expansion of the magnetization to finite order. The leading term in this expansion goes as $T^{3 / 2}$ and is produced by the linear spin wave theory developed by Bloch. The leading term caused by dynamical spin wave interactions is of order $\mathrm{T}^{4}$ which implies that even the dynamical interaction is small at low $T$. Furthermore, at temperatures up to $0.5 T_{c}$ where $T_{c}$ is the curie temperature it was found that the linear, noninteracting spin wave theory discussed by Bloch was quite adequate in describing the behavior of Heisenberg ferromagnets. The physical implications of Dyson's approach have been discussed by Keffer and Loudon ${ }^{9}$ who obtained the $T^{4}$ contribution to the magnetization by assuming that when spin waves are excited at low temperatures they are superimposed onto the instantaneous background of spin wave fluctuation already existing in the system. Dyson's kesults have been approximately reproduced by 0 guch $i^{10}$ who used the Holstein-Primakoff formalism, ${ }^{11}$ in which the spin operators are written in terms of the creation and annihilation operators of the 
harmonic oscillator, in order to obtain the $T^{4}$ contribution to the spontaneoüs magnetization.

The temperature dependence of the spin wave frequency produced by the lowest order nonlinear terms in the isotropic Heisenberg exchange interaction arising from the $\mathrm{H}-\mathrm{P}$ expansion of the spin operators has been studied by M. Bloch. ${ }^{12}$ These lowest terms have the form

$$
\begin{aligned}
& \because H_{\text {ex }}^{(4)}=\frac{1}{2} \vec{k}_{\vec{k} \vec{k}^{\prime}, \vec{k}^{\prime \prime}}\left[J(\vec{k})+J\left(\vec{k}+\vec{k}^{\prime}-\vec{k}^{\prime \prime}\right)-2 J\left(\vec{k}-\vec{k}^{\prime}\right)\right] \\
& \cdot \underset{\vec{k}^{+}}{a_{k^{\prime}}^{+}} \stackrel{a_{k^{\prime \prime}}}{a} \vec{k}+\vec{k}^{\prime}-\vec{k}^{\prime \prime}
\end{aligned}
$$

where $a_{\vec{k}}$ and $a_{\vec{k}}^{+}$are respectively the annihilation and creation operators of a magnon with wave vector $\vec{k}$ and $J(\vec{k})$ is the Fourier transform of the exchange interaction. These spin wave interactions cause a change (renormalization) in the spin wave frequency which for nearest-neighbor interactions is

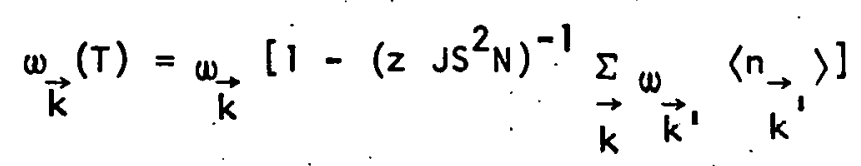

where $z$ is the number of nearest neighbors, and

$$
\omega_{\vec{k}}=2 J z S[\gamma(0)-\gamma(\vec{k})]
$$

is, the noninteracting spin wave frequency and

$$
\gamma(\vec{k})=\frac{1}{2} \sum_{\delta} e^{i \vec{\delta} \cdot \vec{k}} \cdot(\vec{\delta}=n . n \text {. lattice vector })
$$


Also,

$$
\left\langle n_{\vec{k}}\right\rangle=\frac{1}{e^{\operatorname{Bun}_{\vec{k}}(T)}-1}
$$

which is the magnon thermal occupation number. Consequently as the temperature increases the number of excitations increases and the spin wave frequency decreases. M. Bloch has determined that solutions to the coupled equations $(1.2)$ and $(1.5)$ exist even up to temperaiures within a few percent of Curie temperature calculated by the Bethe-Perierls-Weiss method. 13 This nearest neighbor calculation has been extended by Horwitz and Mattis ${ }^{14}$ to the case of non-nearest neighbor interactions. From all of these studies then, it is evident that magnon-magnon interactions remain relatively weak over a broad temperature range; however, all these attempts indicate that the spin wave theory becomes meaningless near and especially above $T_{c}$ in the paramagnetic phase where no long range order exists. Therefore no spin wave excitations should be observed in this temperature region. Recent experimental observations have largely substantiated these theoretical calculations, but some significant deviations have been found.

The specific experiments that will now be discussed in this conneclion investigate the behavior of spin waves via the inelastic scattering of neutrons. The reason for this emphasis lies with the fact that gnetic coupling energies between atoms in solids are typically on the order of a few milli-electron volts and consequently their effects ore conveniently observable in thermal neutron experiments. 
In their study of the spin wave excitations in iron using a triple axis neutron spectrometer, Shirane, Minkiewicz, and. Nathans 15 found that the spin wave modes were well resolved to temperatures very near the Curie temperature, $T_{c} \stackrel{?}{=} 1042^{\circ} \mathrm{K}$. (See Figure 1.) Employing a constant momentum scan where the wave vector $\dot{q} \sim 0.1 \AA^{-1}$, they observed, however, no distinct spin wave peaks in the scattering data when the temperature was raised above $T_{\mathcal{C}}$.

An examination of the spin fluctuations in $\mathrm{Ni}$ near the critical temperature by Minkiewicz, Collins, Nathans and Shirane, ${ }^{16}$. revealed that below $T_{c}$ the magnon peaks were resolved, but above $T_{c}$ they found that the long wavelength $\left(q=0.075 \AA^{-1}\right)$ excitations could be adequately described by the spin diffusion process in which a unit of spin is transferred randomly from one point in the lattice to another with a probability given by the ordinary theory of atomic diffusion. (See Figure 2.)

Recently, Mook, Lynn and Nicklow ${ }^{17}$ have looked at the temperature dependence of short wavelength $\left(q>0.240 \AA^{-1}\right.$ and $\left.E>12 \mathrm{meV}\right)$ sp in waves in nickel and iron. Using a constant energy scan technique which was made necessary because of the steepness of the magnon dispersion curves for $\mathrm{Ni}$ and $\mathrm{Fe}$ at high energies, they observed well resolved excitations ahove. $T_{c}$ that had a rcmarkable similarlty to spin wave modes below the critical temperature. (See Figure 3.) This phenomenon is not, however, limited to metals such as iron and nickel, but has also been observed in the inelastic scattering of neutrons from $\mathrm{RbMnF}_{3},{ }^{18}$ (Figure 4), a magnetic insulator which behaves very much like an idealized Heisenberg 


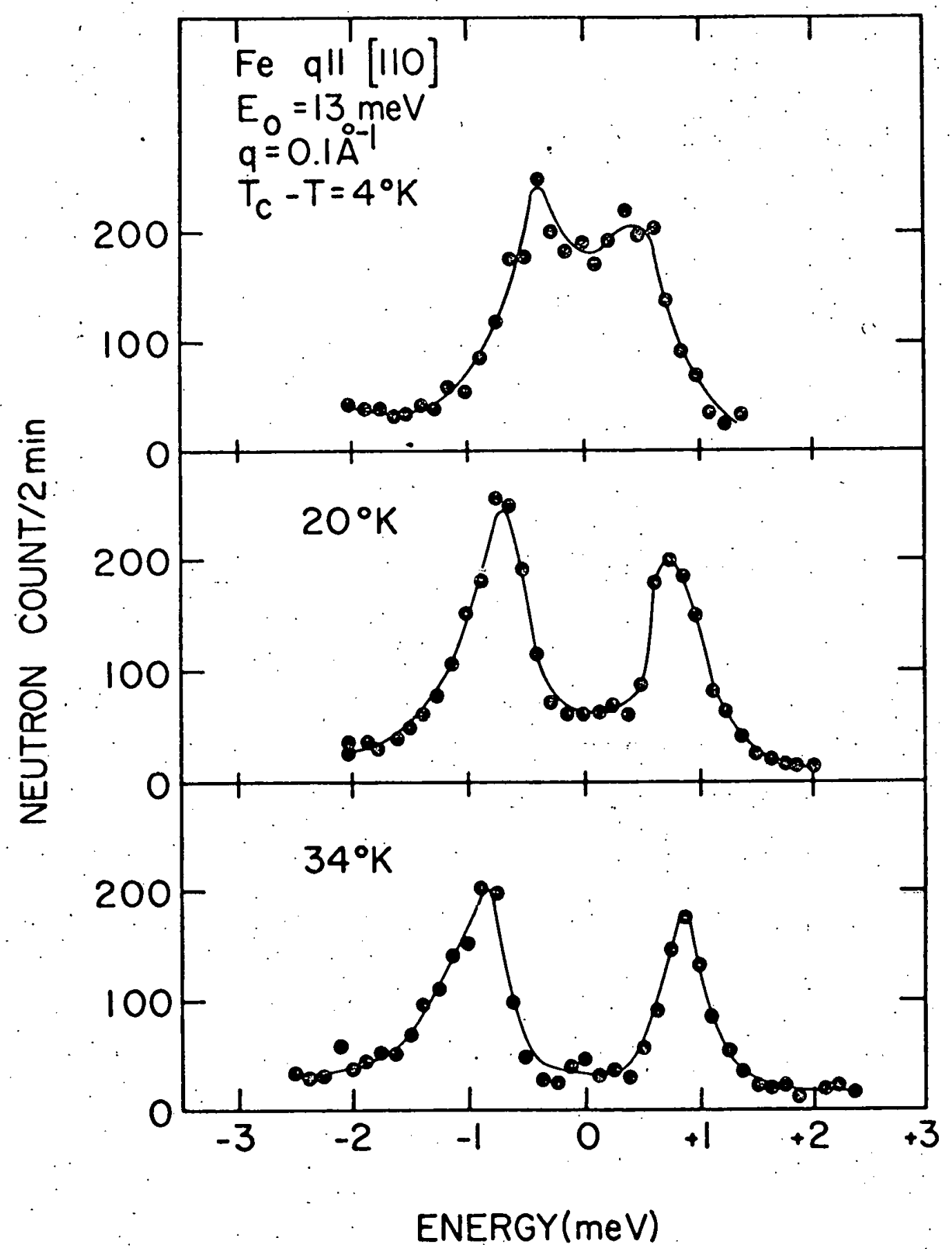

Figure 1. Inelastic neutron scattering intensity for Fe below the Curie temperature $\mathrm{T}_{\mathrm{c}}=1042^{\circ} \mathrm{K}$. Constant momentum scan. 


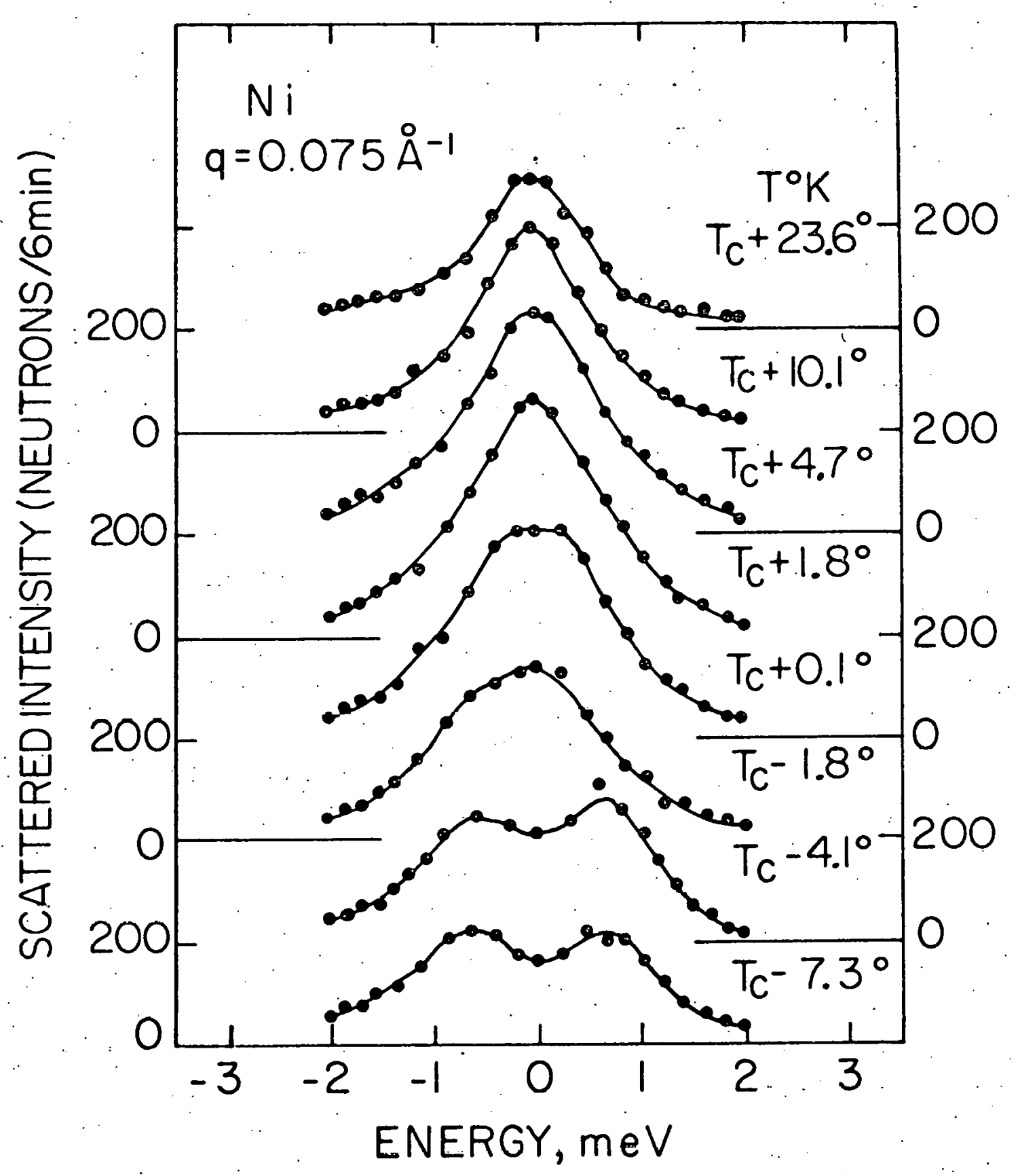

Figure 2. Constant momentum scan for $\mathrm{Ni}$ above and below $\mathrm{T}_{\mathrm{c}}=631^{\circ} \mathrm{K}$. $\vec{q}$ is in the [111] direction. 


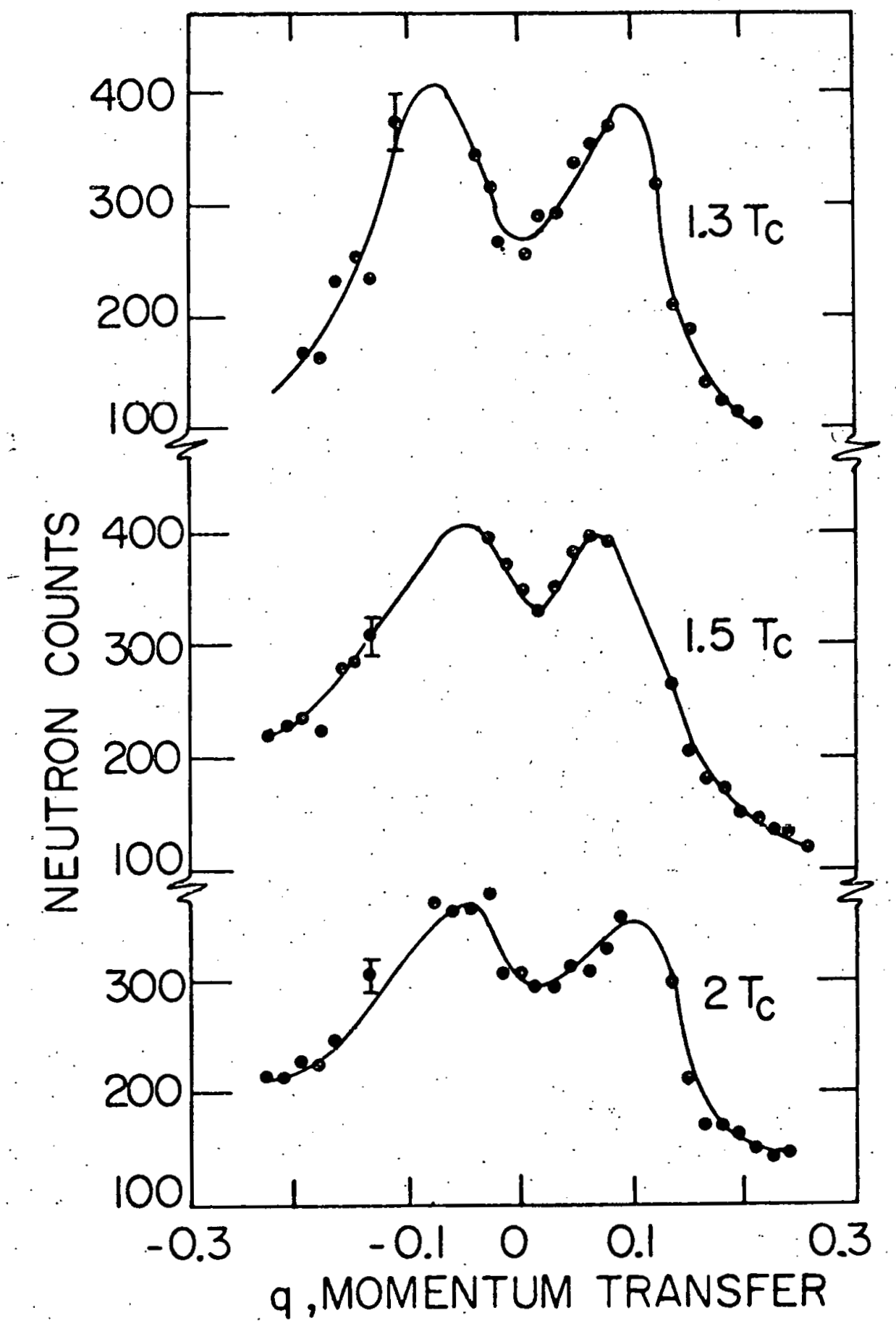

Figure 3. Constant energy scan for $\mathrm{Ni}$ above the Curie temperature about the (11i) reciprocal lattice point when the energy transfer $\Delta E=12.4 \mathrm{meV} . q$ is in zone boundary units of $2 \sqrt{3 / a}$. 


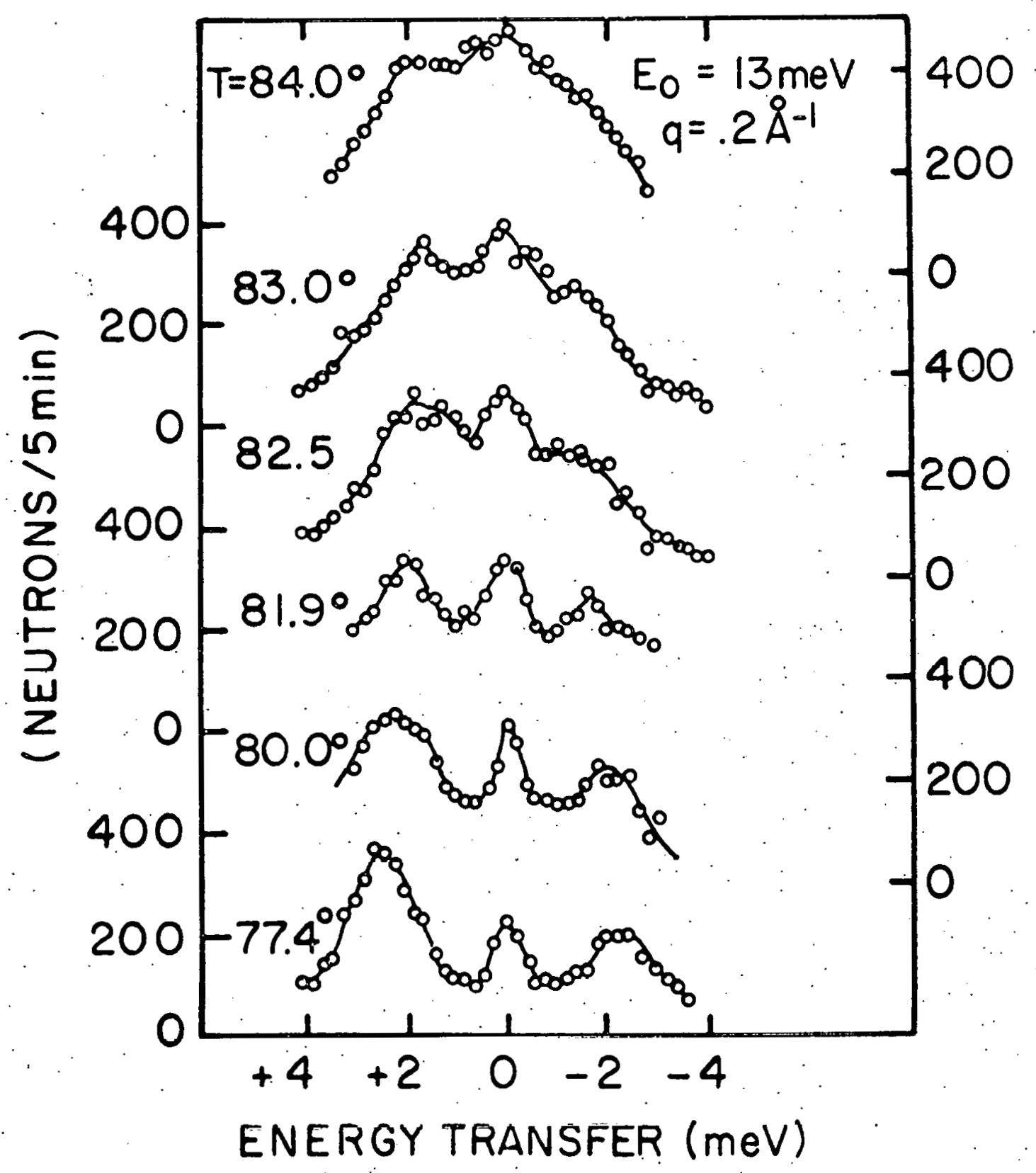

Figure 4. Inelastic scattering from $\mathrm{RbMnF}_{3}$ at an incident energy $E_{0}=13 \mathrm{meV}$. This constant momentum scan was carried out near the ( $\frac{1}{5} \frac{1}{2} \frac{1}{2}$ reflection above and below the Neel temperature $T_{N}=82.6{ }^{\circ} \mathrm{K}$. 
magnet. 19 it is this observation which has motivated us to examine the magnetic excitations of the isotropic Heisenberg model in the paramagnetic phase.

Although we will be primarily interested in the behavior of spin fluctuations in three dimensional magnets such as metals like $\mathrm{Ni}$ and $\mathrm{Fe}$ and insulators like $\mathrm{RbMnF}_{3}$, much work has recently been done in studying the spin dynamics of one dimensional magnets. The experimental investigations have centered on the linear-chain ant iferromagnet $\left(\mathrm{CD}_{3}\right)_{4} \mathrm{NMnCl}_{3}$ (TMMC). 20,21 The reason for the interest in $1-d$ magnetic structures is that the persistence of quasi-spin wave excitations in the paramagnetic regime is more pronounced in the case of systems of lower dimensionality. Furthermore, in $1-d$ systems there is no finite ordering temperature, i.e., long range order exists only at $T=0 .^{22}$ However, experiments on TMMC have demonstrated that there is a highly developed short range order at temperatures near absolute zero. Tomita and Mashiyama ${ }^{23}$ have examined the characteristics of magnon modes in a linear chain of classical spins using the Heisenberg model, and they found fairly good agreement between their calculated inelastic neutron scattering cross section and the experimental data of TMMC.

From all these experiments, then, it. is quite evident that spin wave: theory has a much broader region of applicability than was predicted by the early theories. More recently there have been several attempts to account for the experimentally observed behavior of spin fluctuations in three dimensional systems at high temperatures and near $T_{c}{ }^{\cdot}$ In the 
next section the most salient features of these theories will be discussed along with an examination of their shortcomings. 


\section{REVIEW OF PREV IOUS THEORIES}

The varlous theorles that have been developed which quant Itatively describe spin fluctuations in the paramagnetic phase and their corresponding spectral density fall into two general categories: those which deal directly with the multiple spin time dependent correlation function and attempt to construct a decoupling scheme for this function such as

$$
\begin{aligned}
& \left\langle\underset{\vec{q}_{1}}{\left\langle s_{\vec{q}_{2}}^{-}\right.}(t) \vec{s}_{\overrightarrow{a_{3}}}^{+}(t), \underset{\vec{q}_{3}}{s_{\vec{q}_{4}}^{-}}(0) \vec{s}_{\vec{q}_{4}}^{+}(0)\right\rangle \simeq \delta\left(\vec{q}_{1}+\vec{q}_{4}\right) \delta\left(\vec{q}_{2}+\vec{q}_{3}\right) \beta^{-1}
\end{aligned}
$$

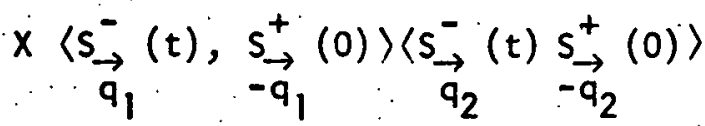

and those which chararterize the spectral shape of the associatcd excitations in terms of its lowest order moments, namely the zeroth, second, and fourth moments, and relates these moments to higher order static correlation functions.

One of the earliest discussions of this problem was given by Marshal1 24 in which he described very qualitatively the temperature dependence of spin waves. In his work it was noted that just above $T_{c}$ when the momentum transfer of the scattered neutrons is large, short wavelength spin fluctuations are excited which behave very much like magnon modes at low temperatures. These excitations were called remnant spin waves. Within thls framework some of the experimental observations 
can be readily understood: when neutrons are scattered at large wave vectors just above $T_{c}$ where $q>x$, the inverse correlation length, then remnant spin waves should be observed. However, in the case of scattering at smaller wave vectors where $q<x$ the excitations span many clusters of spins that are correlated in different directions and consequently must behave very differently from spin waves. Although this picture satisfies the experimental observations in a qualitative way, the previous theoretical attempts to account for the observed spectral shape near the critical point run into difficulties which will be evident in the following discussion.

The first extensive theoretical investigation of spin fluctuations above the critical temperature was done by Resibois and De Leener ${ }^{25-28}$. who concentrated on an approximate evaluation of the spin autocorrelation function

$$
\Gamma_{a b}^{\alpha \beta}(t)=\left\langle s_{a}^{\alpha}(t) s_{b}^{\beta}(0)\right\rangle
$$

which plays a central role in neutron inelastic scattering measurements. In this expression $s_{a}^{\alpha}(t)$ is the Heisenberg representation of the $\alpha$ th component of the spin operator located at the lattice site a, and $\langle.$. is an average over an equilibrium cannonical ensemble. Using a localized spin model we can write the autocorrelation function in the following manner

$$
\Gamma_{a b}^{\alpha \beta}(t)=\sum_{m^{\prime} m^{\prime}}\left\langle m\left|s_{a}^{\alpha}\right| m^{\prime}\right\rangle\left\langle m^{\prime}\left|p^{\beta}(t \mid b)\right| m\right\rangle,
$$



In extending their theory to finite temperatures in the neighborhood of the critical temperature the following non-Markoffian kinetic equation for the autocorrelation function $\Gamma_{a b}^{\alpha \beta}(t)$ was derived:

$$
\frac{\partial}{\partial t}\left[\tilde{\Gamma}_{q}(t)\right]=\int_{0}^{t} \tilde{G}_{q}\left(t-t^{\prime} \mid \tilde{\Gamma}_{q^{\prime}}\right) \tilde{\Gamma}_{q}\left(t^{\prime}\right) d t^{\prime},
$$

where $\tilde{\Gamma}_{q}(t)$ is the space Fourier transform of $\Gamma_{a b}^{\alpha \beta}(t)$ and $\tilde{G}_{q}\left(t \mid \tilde{\Gamma}_{q}\right)$ is the Fourier transform of an infinite series of terms whose form is determined by a diagrammatical analysis which was discussed in detail. Only the lowest order "bubble" diagrams were kept in order to obtain an approximate expression for $\check{\Gamma}_{q}(t)$.

Near the critical point the shape of the frequency spectrum near $T_{c}$, which is directly connected with the observed neutron scattering cross section, is seen in Figure 5 , where $\gamma$ is the spectral shape functinn

$$
\gamma(\Omega)=1 /\left[\Omega^{2}+\left(1-\epsilon \delta^{2}\right)^{2}\right]
$$

and $\Omega$ is a dimensionless frequency

$$
\Omega=\omega\left(\alpha q^{\frac{5}{2}}\right)^{-1}
$$

with $\epsilon$ and $\alpha$ as fitting parameters.

One of the major short comings of this theory is that the whole approach is only valid in the Weiss limit where there are an infinite number of neighbors; however in a realistic situation $z=6$ or 8 , 
16 
and as we shall see the effects of short range order on the observed neutron cross section become dominant when the number of neighbors within the range of the exchange interaction is finite and small.

Another approach to the analysis of time dependent spin correlations at high temperatures was presented by Blume and Hubbard. ${ }^{29}$ The basic physical idea behind their mathematical calculation is that each spin interacts with a randomly varying effective field which is produced by the neighboring fluctuating spins. The correlation functions $c_{q}=\left\langle s_{-q}^{z} s_{q}^{2}(t)\right\rangle$ are evaluated indirectly through the use of the relaxation function $\left\{s_{-q}^{2}, s_{q}^{2}(t)\right\}$ where by definition:

$$
\{A, B(t)\}=\int_{0}^{\beta}\left\langle e^{\lambda H} A e^{-\lambda H} B(t)\right\rangle d \lambda \text {. }
$$

This function describes the relaxation of the variable $B$ for $t>0$ when a perturbation which is proportional to $A$ is switched on adiabatically, between $t=-\infty$ and $t=0$ and then is suddenly turned off at $t=0$. Then when $T \rightarrow \infty$

$$
\frac{\left\langle s_{-q}^{z} s_{q}^{z}(t)\right\rangle}{\left\langle s_{-q}^{z} s_{q}^{z}\right\rangle}=\frac{\left\{s_{-q^{2}}^{z} s_{-q}^{z}(t)\right\}}{\left\{s_{-q^{2}}^{z}, s_{q}^{z}\right\}}=F_{q}(t)
$$

where $(\ldots)$ denotes an average over an equilibrium canonical ensemble, and thus

$$
C_{q}(t)=\frac{1}{3} N^{-1} s(s+1) F_{q}(t)
$$


Kubo's formalism ${ }^{30}$ is then used to show that

$$
F_{q}(t)=\frac{\left\langle\delta \cdot s_{q}^{z}(t)\right\rangle}{\left\langle\delta s_{q}^{z}\right\rangle}
$$

where $0 s_{q}^{z}(t)$ represents the change in $s_{q}^{z}(t)$ due to the perturbation $s_{-q}^{z}$. Now in order to find $F_{q}(t)$ a formal time-order solution is obtained for the equation of motion for $\operatorname{dis}_{q}^{z}(t)$ :

$$
\delta \dot{s}_{q^{\prime}}{ }^{2}=\sum_{q^{\prime \prime}} j\left(\vec{q}^{\prime}, \vec{q}^{\prime \prime}\right) s^{+}{\overrightarrow{q^{\prime}}}_{-\vec{q}^{\prime \prime}} \delta s_{\vec{q}^{\prime \prime}}^{-}
$$

where

$$
J\left(\vec{q}^{\prime}, \vec{q}^{\prime \prime}\right)=J\left(\vec{q}^{\prime}\right)-J\left(\vec{q}^{\prime \prime}\right)
$$

The random effective magnetic field is then associated with the term $J\left(\vec{q}^{\prime}, \vec{q}^{\prime \prime}\right) S^{+} \vec{q}^{\prime}-\vec{q}^{\prime \prime}$. The solution of $F_{q}(t)$ consequently, involves the evaluation of time dependent multiple spin correlation functions. The principal approximation made is to neglect the detailed correlations between three or more.spins. This approximation is not too bad at high temperatures where it leads to errors of $O(1 / z)$ where $z$ is the number of magnetic ions lying within the range of the exchange interaction. The result of this method, valid for general times in the high temperature limit. $(T \rightarrow \infty)$, is seen in Figure 6 . It is evident from these plots that there is no indication of propagating modes-- only one that is 


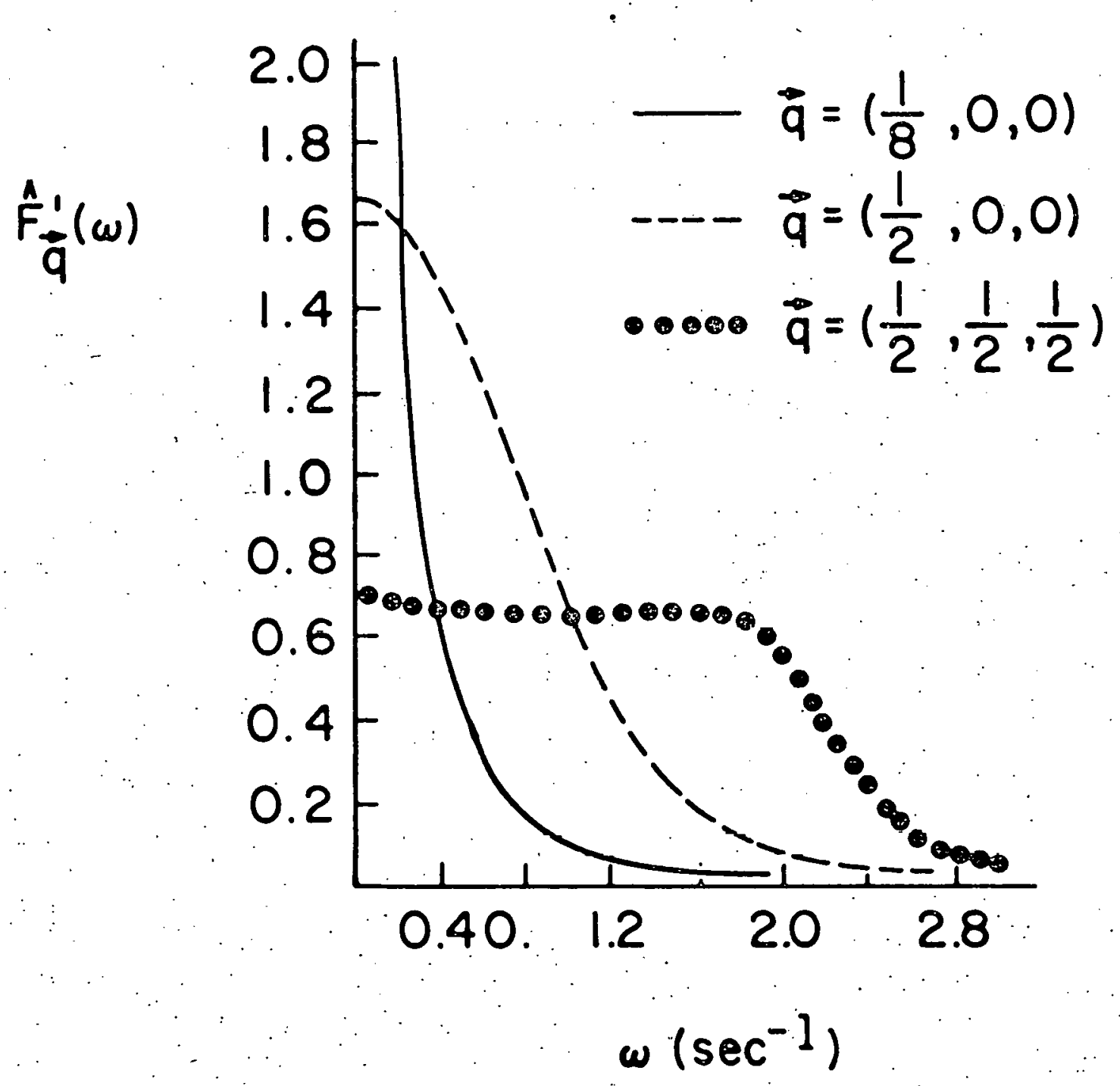

Figure 6 Fourier transform of the relaxation shape function for a simple cubic lattice obtained by, Blume and Hubbard for various wave vectors, $\vec{q}$, where $\vec{q}$ is in units of $2 \pi / a$. 

which is closely related to the spin autocorrelation function $\Gamma_{a b}(t)$ used by Resibois and De Leener. Actually $\underset{\alpha}{\Sigma} \Gamma_{a b}^{\infty \alpha \alpha}(t)$ is equivalent to $U_{\vec{R}}(t)$ to within a factor $s(S+1)$.

The moments of $\rho_{x}(11)$ are

$$
\left\langle(1)^{2 n}\right\rangle_{x}=\frac{\left.\int \rho_{n}(u) u\right)^{2 n} d \omega}{\int \rho_{n}(v) d u} .
$$

The expressions for $\left\langle()^{2}\right\rangle_{x}$ and $\left\langle()^{4}\right\rangle_{x}$ are valid only in, the high temperature limit and for polycrystals. The wave vector dependence of the second and fourth moments indicated that for small wave vectors $(x \rightarrow 0)$ the spectral density $\rho_{x}(\omega)$ can be approximated by a truncated Lorentzian. At larger wave vectors where $x \geq \pi / b$ where $b$ is the nearest neighbor distance the shape of $p_{x}(\omega)$ is more closely given by a Gaussian function.

A preliminary attempt to determine whether or not propagating modes exist in a Heisenberg paramagnet was carried out by Bennett, 32 who calculated the lowest order moments of an effective frequericy spectrum assumed to have no delta-furiclion of frequency terms, $\delta(5)$, and to have a smooth monotonic behavior. It is noted that the validity of this approach depends on the validity of the diffusion equation for the magnetization in the hydrodynamic regime ${ }^{33}$ where $q \ll x$, the long wavelength region. Then the multiplespin time dependent correlation functions appearing in the calculation are decoupled in the following way: 


$$
\left\langle s_{i}^{z} s_{j}^{+} s_{k}^{z} s_{l}^{-}\right\rangle \approx\left\langle s_{i}^{z} s_{k}^{z}\right\rangle\left\langle s_{j}^{+} s_{l}^{-}\right\rangle
$$

and

$$
\left\langle s_{i}^{+} s_{j}^{-} s_{k}^{+} s_{l}^{-}\right\rangle \approx\left\langle s_{i}^{+} s_{j}^{-}\right\rangle\left\langle s_{k}^{+} s_{l}^{-}\right\rangle+\left\langle s_{i}^{+} s_{l}^{-}\right\rangle\left\langle s_{k}^{+} s_{j}^{-}\right\rangle
$$

When this approximation is used to simplify the expressions for the second and fourth moments Bennett finds that the criterion for the existence of propagating modes when $q>\xi^{-1}$ where $\xi$ is the spin correlation length is

$$
R(\vec{q})=\left\langle w^{2}\right\rangle_{q}^{2} /\left\langle\left(w^{2}-\left\langle w_{q}^{2}\right\rangle\right)^{2}\right\rangle_{q} \gg 1
$$

When this inequality is satisfied high frequency modes exist; however when $q<\xi^{-1}$

$$
R(\overrightarrow{4})<1
$$

and diffuse modes dominate. The fact that Bennett's calculations are carried out in the hydrodynamic regime is one of the most prominent shortcomings of his theory. The reason for this is found in Halperin and Hohenberg's discussion ${ }^{33}$ of the critical and hydrodynamic regions where the latter is defined to occur when $q \ll x$. However, as will be shown below, the propagating modes observed by Mook et al. ${ }^{17}$ in $\mathrm{Ni}$ above 
the critical temperature occur when $q>x$, the inverse correlation length. Consequently, the decoupling scheme used by Bennett is in question. It is apparent from his work that no attempt was made to consider the possible contribution of short range order to the shape of the frequency spectrum above $T_{c}$.

of greater interest is the work done by Tomita and Tomita ${ }^{34}$ in which theoretical line shapes are obtained for the relaxation shape function. These shapes are then compared with the neutron inelastic magnetic scattering from $\mathrm{RbMnF}_{3}{ }^{18}$. The second and fourth moments of the relaxation function are calculated assuming nearest-neighbor interactions only. The general forms of these moments are

$$
\left\langle\omega^{2}\right\rangle_{\vec{k}}=\frac{[1-\lambda(\vec{k})]}{n^{2} x^{22}(\vec{k})} \frac{1}{N} \underset{q}{q} J(\vec{q})\left\langle s^{+}(-\vec{q}) s^{-}(\vec{q})\right\rangle,
$$

where

$$
\lambda(\vec{k})=\frac{1}{z} \frac{\sum}{a} e^{i \vec{k} \cdot \vec{a}}
$$

and

$$
J(\vec{q})=J \underset{\vec{a}}{\sum^{\mid \vec{q} \cdot \vec{a}}}
$$

In which $z$ is the number of nearest-neighbors, $\vec{a}$ is the nearest-neighbor position vector, $J$ is the nearest-neighbor exchange interaction, and 
$x^{z Z}(\vec{k})$ is the longitudinal wave vector dependent susceptibility. at small $k$

$$
\left\langle\omega^{4}\right\rangle_{\vec{k}}=\frac{k^{2}}{\hbar^{4} x^{22}(\vec{k})} \times\{\text { set of 4-spin correlation functions\}. }
$$

Then the high temperature $(T \rightarrow \infty)$, finite temperature $\left(T>T_{c}\right)$, and low temperature $(T \rightarrow 0)$ behavior of these moments is discussed. At finite temperatures the random phase approximation (R.P.A.) form of the two-spin correlation function,

$$
\left\langle s^{z}(\overrightarrow{-q}) s^{z}(\vec{q})\right\rangle=\frac{N k_{B} T}{x_{0}^{-1}+[J(0)-J(\vec{q})]}
$$

where $x$ is the paramagnetic susceptibility, is used in finding explicit expressions for $\left\langle\omega{ }^{2}\right\rangle_{q}$ and $\left\langle\omega{ }^{4} r_{q}\right.$. Also for $\left.T\right\rangle T_{c}$ the calculation of the fourth moment is made tractable by expressing the four-spin correlation function in terms of a product of two spin correlation functions. Bennett has also employed this same type of approximation which is valid in the high temperature limit where the number of neighbors $z$ is large since the error introduced is of the order of $1 / 2$. In comparing the temperature dependence of the second moment to that of the fourth moment, Tomita and Tomita find that the fourth moment is more sensitive to fluctuations in the spin system as the temperature changes than the 
second moment due to the higher order multi-spin correlations contained in $\left\langle\omega^{4}\right\rangle \vec{q}$

In order to obtain their spectral line shapes, Mori's well-known continued fraction expansion ${ }^{35}$ is used in evaluating the canonical correlation function

$$
\psi(\vec{k}, \omega)=\int_{0}^{\infty} d t e^{-i \omega t} \frac{R(\vec{k}, t)}{R(\vec{k}, 0)}
$$

where $R(\vec{k}, t)$ is the relaxation function. This continued fraction expansion ${ }^{36}$ may be written as

$$
\left.\psi(\vec{k}, \omega)=\Gamma i \omega+a_{1}^{2}(\vec{k}) f\left(\omega ;\left\{b^{2}(\vec{k})\right\}\right)\right]^{-1}
$$

where

$$
f\left(\omega ;\left\{b^{2}(\vec{k})\right\}\right)=\left[i \omega\left(a_{2}^{2}(\vec{k}) / i \omega+\cdot \cdot \cdot\right)\right]^{-1},
$$

and

$$
\begin{aligned}
& a_{1}^{2}(\vec{k})=\left\langle\omega^{2}\right\rangle_{\vec{k}} \equiv a^{2}(\vec{k}) \\
& a_{2}^{2}(\vec{k})=\frac{\left\{\left\langle\omega^{4}\right\rangle_{\vec{k}}-\left\langle\omega^{2}\right\rangle_{\vec{k}}^{2}\right\}}{\left\langle\omega^{2}\right\rangle_{\vec{k}}} \equiv b^{2}(\vec{k})
\end{aligned}
$$

[Note that $R(\vec{k}, t)$ is equivalent to $B$ lume and Hubbard's $C_{\vec{k}}(t)$ and that $\psi(\vec{k}, \omega)$ is just the Laplace transform of their normalized spectral shape 
function $\left.F_{\vec{k}}(t).\right]$ Here $f\left(w ;\left\{b^{2}(\vec{k})\right\}\right)$ is the torque correlation function in which $\left\{\vec{b}^{2}(\vec{k})\right\}$ corresponds to the $\operatorname{set}\left\{a_{2}^{2}(\vec{k}), a_{3}^{2}(\vec{k}), \ldots.\right\}$. Then it is assumed that the torque correlation spectrum can be characterized by only $a_{2}^{2}(\vec{k})$ and further that the shape of this spectrum can be simply described by a Gaussian function. The total frequency spectrum obtained can be seen in Figure 7. Although there are peaks in the theoretical line shape calculated by Tomita and Tomita, serious discrepancies exist between their shape function and the observed inelastic neutron scattering data for RbMnF $3^{\circ}$ (See Figure $8_{.}$) Two problems arise with their approach: first, the second and fourth moments are evaluated through the use of a decoupling scheme that is valid only at high temperatures. Another difficulty concerns the particular truncation procedure used which introduces mathematically a two peaked function into the theory. It would be more meaningful if the derivation of these peaks could be based on the nature of the physical processes which govern the behavior of the spin system above the critical point such as the existence of short range order.

Recently Lovesey and Meserve 37 have employed Mori's continued fraction expansion of the relaxation spectral shape function in analyzing the frequency spectrum of the inelastic neutron scattering cross section. Here again a truncation procedure is developed in order to approximate the relaxation function, however the expansion is carried to one order farther than Tumita and Tomita's work so that a three pole approximation is constructed. The second and fourth moments involved in this calculation are evaluated at infinite temperature 38 and at finite temperatures 


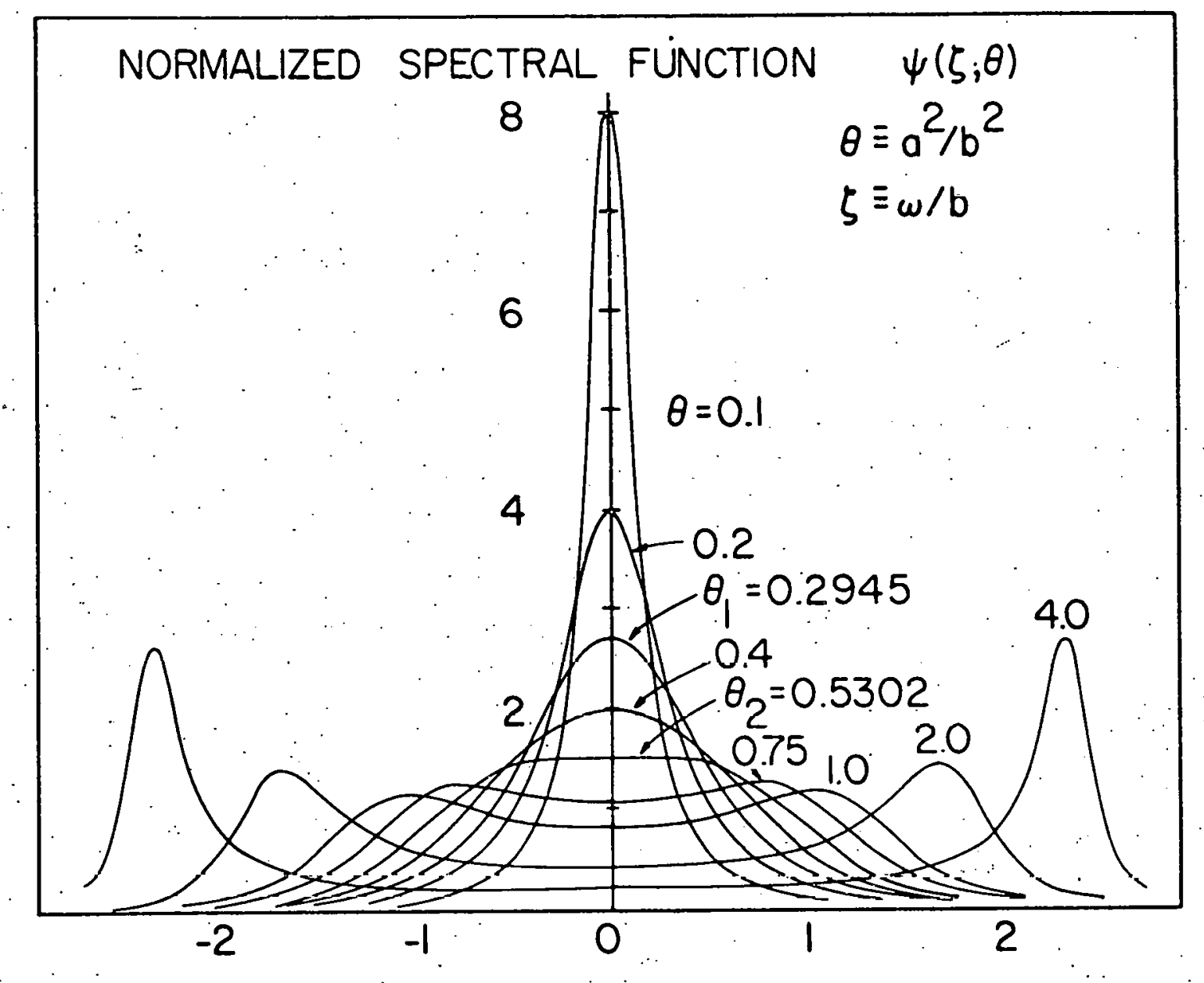

FREQUENCY $\zeta$

Figure.7. Spectral function obtained by Tomita and Tomita. At $\theta_{1}$ the poles of $\psi$ become complex and when $\theta>\theta_{2}$ peaks may be discerned. 


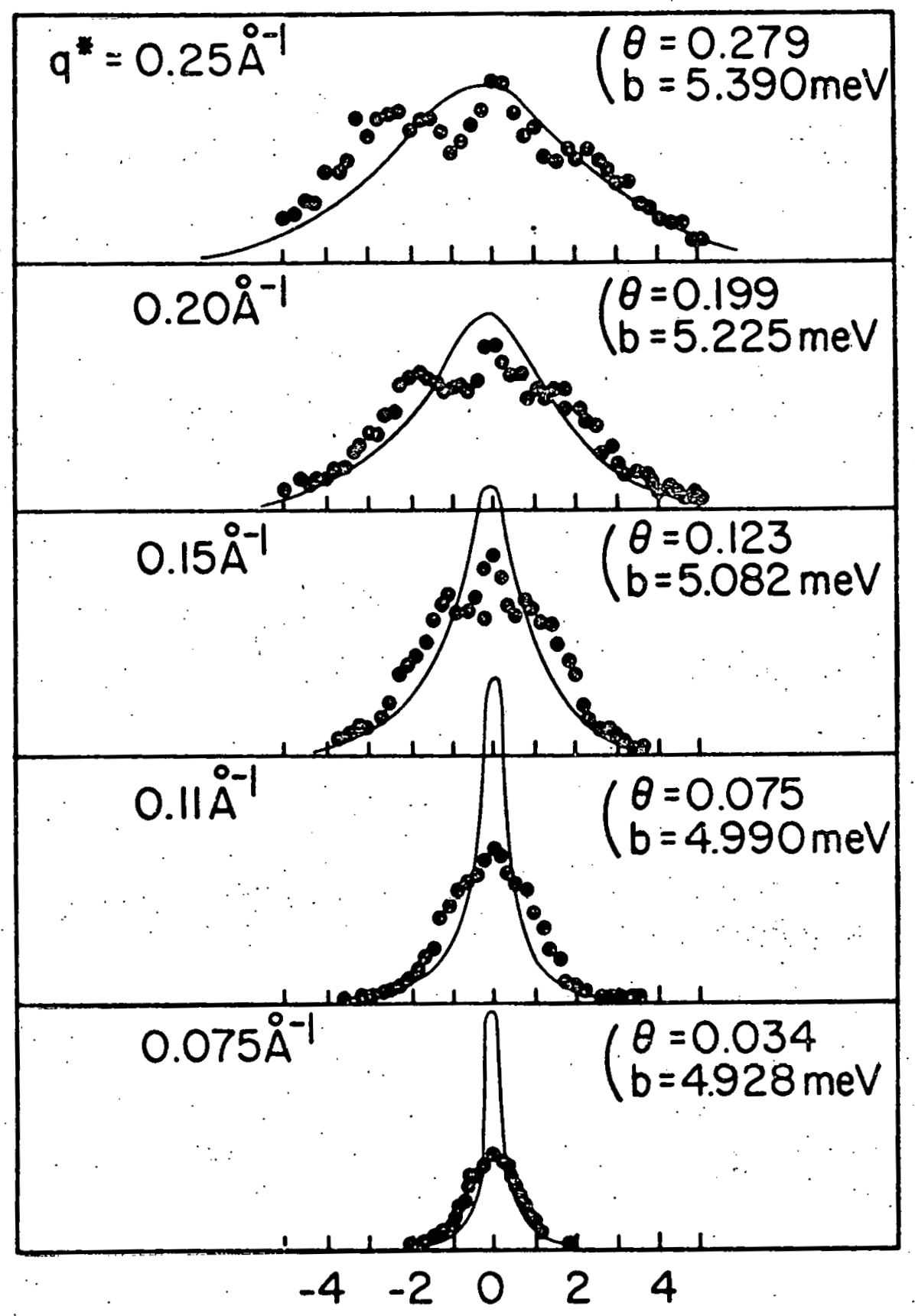

ENERGY TRANSFER $\omega(\mathrm{meV})$

Figure 8. Comparison between the theoretical lines obtained by Tomita and Tomita and inelastic neutron scattering data for RbMnF 3 . $q^{*}$ is measured along the [111] direction. 
$T>T_{c}$ where the four spin correlation functions contained in the expression for the fourth moment are approximated in terms of two-spin correlation functions in a manner quite similar to the decoupling scheme used by Tomita and Tomita. The agreement between this method and the experimental data ${ }^{18}$ obtained for $\mathrm{RbMnF}_{3}$ is fairly good. However the same objections that were raised there also apply in this discussion: the truncation procedure automatically determines a function which has the desired number of poles in it. There is no attempt to determine from the physics of the system why the particular structure in the spectrum is observed, i.e. the possible effects of short range order near $T_{c}$ are again not explicitly considered. Furthermore, a honphysical peak in the theoretical line shape occurs at $T=\infty$ when $\vec{k}>\left(\frac{1}{3}, \frac{1}{2}, \frac{1}{2}\right)$.

One of the most detailed examinations of the moment expansion method was presented by Reiter 39 in his study of spin fluctuations in Heisenberg paramagnets. Reiter obtains an infinite series expansion of the relaxation function in terms of the moments of the spectral density. The relaxation of the $q$ th component of the magnetization in the absence of any externally applied fields is given by

$$
\Sigma(\vec{q}, t)=\left\langle s^{2}(\vec{q}, t), s^{z}(-\vec{q}, 0)\right)=\left\langle s^{z}(-\vec{q}, 0) \mid s^{z}(\vec{q}, t)\right\rangle .
$$

where

$$
(A, B) \equiv\{A, B\}
$$


which is defined in Eq. $(11.10)$, and where

$$
\langle A \mid B\rangle=\left\{A^{+}, B\right\}
$$

Thus $\Sigma(\vec{q}, t)$ is the unnormalized form of the shape function $F_{q}(t)$ which is used by Blume and Hubbard. [See Eq. (11.11)].

In terms of its moments

$$
\Sigma(\vec{q}, t)=\sum_{n=0}^{\infty} \frac{(-i t)^{n}}{n !} \times(\vec{q}, 0)\left(w^{n}\right\rangle^{\cdot}
$$

Here $x(\vec{q}, 0)$ is the isothermal response of the magnetization to a magnetic field of strength $\left(g \mu_{B}\right)^{-1}$ and wave vector $\vec{q}$, where

$$
\left\langle\omega^{n}\right\rangle_{q}=\frac{\left\langle s^{z}(\vec{q})\left|L^{n}\right| s^{z}(\vec{q})\right\rangle}{x(\vec{q}, 0)},
$$

in which $L$ is the Lionville operator of the system:

$$
\text { LO }=\frac{1}{n}[0, H]
$$

Diagrammatic expansions for theșe moments are developed for infinite temperature and for finite temperatures above $T_{c}{ }^{\cdot}$ When $T>T_{c}$ the second moment $\left\langle\omega^{2}\right\rangle_{q}$ and its fluctuation $\left\langle\Omega_{q}^{4}\right\rangle=\left\langle\left(\omega^{2}-\left\langle\omega^{2}\right\rangle_{q}\right)^{2}\right\rangle_{q}$ become 


$$
\left\langle\omega^{2}\right\rangle_{q}=\frac{1}{3} s(s+1) N^{-1} \cdot \underset{\vec{q}^{\prime}}{\Sigma}\left[V\left(\vec{q}^{\prime}\right)-V\left(\vec{q}-\vec{q}^{\prime}\right)\right]^{2} \rho\left(\overrightarrow{q^{\prime}}\right) \rho\left(\vec{q}-\vec{q}^{\prime}\right) \rho(\vec{q})^{-1},
$$

and

$$
\begin{aligned}
\left\langle\Omega^{4}\right\rangle_{q}=\frac{2}{3}[S(s+1)]^{2} N^{-2} \underset{\vec{q} !}{\sum} \vec{q}_{q^{\prime \prime}}\left[V\left(\vec{q}-\vec{q}^{\prime}\right)\right]\left[V\left(\vec{q}^{\prime \prime}\right)-V\left(\vec{q}-\vec{q}^{\prime}-\vec{q}^{\prime \prime}\right)\right] \\
\quad\left[V\left(\vec{q}^{\prime}+\vec{q}^{\prime \prime}\right)-V\left(\vec{q}-\vec{q}^{\prime}-\vec{q}^{\prime \prime}\right)\right]\left[V\left(\vec{q}^{\prime \prime}\right)-V\left(\vec{q}^{\prime}\right)\right] p(\vec{q}) p\left(\vec{q}^{\prime \prime}\right) \\
\therefore\left(\vec{q}-\vec{q}^{\prime}-\vec{q}^{\prime \prime}\right) p(\vec{q})^{-1},
\end{aligned}
$$

where $\rho(\vec{q})$ is the equilibrium spin pair correlation function $p(\vec{q}) \propto\left\langle s_{\vec{q}}^{z} s_{-\vec{q}}^{z}\right\rangle$. When the mament expansion is carried to finite (lowest) order the expression obtained for the relaxation function is valid. for short times only.

Now in order to account for the details observed in the spectral density associated with the neutron inelastic scattering cross section, Reiter 40 obtains a set of kinetic equations for the two spin correlation functions $\left\langle s^{-}\left(\vec{q}_{1}\right) s^{+}\left(\vec{q}_{2}\right)\right\rangle$ and $\left\langle s^{z}\left(\vec{q}_{1}\right) s^{z}\left(\vec{q}_{2}\right)\right\rangle$. These kinetic equations are then used to determine $\Sigma(\vec{q}, t)$ and $\Gamma\left(\vec{q}_{1}, \vec{q}_{2}, t\right)$ which describes the decay of a fluctuation of wave vector $\vec{q}=\vec{q}_{1}+\vec{q}_{2}$ into two fluctuations of wave vectors $\vec{q}_{1}$ and $\vec{q}_{2}$. The expressions for $\left\langle\omega^{2}\right\rangle_{\vec{q}}$ and $\left\langle\Omega^{4}\right\rangle_{\vec{q}}$ are then obtained from the leading terms of series expansion in powers of $\left(\frac{1}{z}\right)$. for $\Gamma\left(\vec{q}_{1}, \vec{q}_{2}, z\right)$, the Laplace transform of $\Gamma\left(\vec{q}_{1}, \vec{q}_{2}, t\right)$, and they agree completely with those derived from the diagramatic expansion of the moments. However the physical implications of the approximations used in obtaining 
the kinetic equations are much more apparent than those used in the diagram approach. In particular the coupled equations of motions for $s_{z}\left(\vec{q}_{1}\right), s^{+}\left(\vec{q}_{1}\right) s^{-}\left(\vec{q}_{2}\right), s^{z}\left(\vec{q}_{1}\right) s^{z}\left(\vec{q}_{2}\right)$ and $s^{-}\left(\vec{q}_{1}\right) s^{z}\left(\vec{q}_{2}\right) s^{+}\left(\vec{q}_{3}\right)$ are closed by decoupling the four-spin correlation functions in the equation of motion for $\left\langle s^{-}\left(\vec{q}_{1}\right) s^{z}\left(\vec{q}_{2}\right) s^{+}\left(\vec{q}_{3}\right)\right\rangle$ as foll lows:

$$
\begin{aligned}
& \left\langle s^{-}\left(\vec{q}_{1}\right) s^{z}\left(\vec{q}_{2}\right) s^{z}\left(\vec{q}_{3}\right) s^{+}\left(\vec{q}_{4}\right)\right\rangle \cong \\
& \left.\because \quad s^{z}\left(\vec{q}_{2}\right) s^{z}\left(\vec{q}_{3}\right)\right\rangle\left\langle s^{-}\left(\vec{q}_{1}\right) s^{+}\left(\vec{q}_{4}\right)\right\rangle,
\end{aligned}
$$

and

$$
\begin{array}{r}
\left\langle s^{-}\left(\vec{q}_{1}\right) s^{-}\left(\vec{q}_{2}\right) s^{+}\left(\vec{q}_{3}\right) s^{+}\left(\vec{q}_{4}\right)\right\rangle \cong \\
\quad\left\langle s^{-}\left(\vec{q}_{1}\right) s^{+}\left(\vec{q}_{3}\right)\right\rangle\left\langle s^{-}\left(\vec{q}_{2}\right) s^{+}\left(\vec{q}_{4}\right)\right\rangle+ \\
\therefore \quad\left\langle s^{-}\left(\vec{q}_{1}\right) s^{+}\left(\vec{q}_{4}\right)\right\rangle\left\langle s^{-}\left(\vec{q}_{2}\right) s^{+}\left(\vec{q}_{3}\right)\right\rangle .
\end{array}
$$

As noted by Reiter this decoupling method neglects those correlations which are nonvanishing when all the spins in $\langle.$. .) are "close"l to one another. This is valid when the range of the exchange interaction is much larger than the range of the four-spin cumulant correlation function. The error introduced in $O(1 / c)$, where $c$ is the number of spins within the range of the exchange interaction. It is precisely these terms which Reiter neglects which will be found to become important near T. 
The high temperature approximation for $\Gamma\left(\vec{q}_{1}, \vec{q}_{2}, z\right)$ which is used to obtain the overall shape of the spectral density is

$$
\Gamma\left(q_{1}, q_{2}, z\right)=\left\{N^{-\frac{1}{2}} \frac{2}{3} s(s+1) \frac{\left[p\left(\vec{q}_{2}\right)-p\left(\vec{q}_{1}\right)\right]}{\rho\left(\vec{q}_{1}+\vec{q}_{2}\right)}\right\} / \nu(z)
$$

where $\nu(z)$ is determined in such a manner that the correct forms of $\left\langle\omega^{2}\right\rangle_{q}$ and $\left\langle\Omega^{4}\right\rangle_{q}$ are maintained. This constant relaxation time approximation is then used to obtain theoretical line shapes which are compared with the neutron inelastic scattering data for $\mathrm{RbMnF}_{3}{ }^{18}$ it is evident that Reiter's approach does describe the shape of the frequency spectrum at high temperatures very adequately, however at low temperatures near $T_{c}$ there are discrepancies especially near $\omega=0$.

The shortcomings that are inherent in the theoretical work deal ing with spin fluctuations in three dimensional Heisenberg ferromagnets are not however present in the studies of one dimensional (1d) Heisenberg systems. Fisher 41 has obtained exact solutions for both the partition function and the spin pair correlation function for a one dimensional Heisenberg ferromagnet and antiferromagnet in the case where $S \rightarrow \infty$. In this limit the classical spin Hamiltonian is obtained i.e., the spin operators in the Hamiltonian commute. Fisher's results have been extended by Tomita and Mashiyama who have rigorously calculated exact expressions for the second, fourth and sixth moments of the time-dependent spin correlation functions for a Heisenberg Id chain of classical spins with nearest-neighbor interactions only. Mori's continued fraction 
expansion was then used to obtain the spectral shape of the inelastic scattering cross section. Their calculated result compares quite favorably to the inelastic neutron scattoring dota of THMC obtained by Hutchings et al. ${ }^{20}$ who determined that this system is adequately described by the classical Heisenberg's ld antiferromagnet with nearest neighbor interactions when $T \gtrsim 1.1{ }^{\circ}$. In both the theory and experiment, welldefined spin wave modes were observed in the paramagnetic region. These short wavelength spin wave excltatlons occur tocause of the existence of a highly-developed short range order just above the critical point which in the case of a ld ferromagnet is $T_{C}-0^{\circ} \mathrm{K}$.

- Recently Mclean and 81 ume 42 have studied the spin dynamics at finite temperature of Id Heisenberg magnetlc chains involving spins of arbitrary size. They examined both the long wavelength and the short wavelength spin fluctuation in this system using Blume and Hubbard's technique which has been discussed above. The existence of short range order was emplicitly allowed for through the introduction of a local order parameter $\Delta$ where $\Delta$ is taken to be the square root of the nearneighbor correlation. When this parameter was included in their calculation it was found that the short wavelength excltations, $q \gg>^{1} / l_{c}$, where $l_{c}$ is the correlation length, are spin-wave-like and that the long wavelength fluctuations, $q<i / \ell_{c}$ ore diffusive in nature and are associated with variatlons in the local order parameter: Again the comparison between the experimental dats of TMMC determined by Hutchings et al. ${ }^{20}$ and their theoretical scottering function is quite good. 
The major point of this whole discussion is that none of the existing theories adequately describes the structure observed in the inelastic neutron scattering data near $T_{c}$ at large wave vectors except in one dimension. In all the $3 d$ theories discussed above one of the major shortcomings was the way in which the multiple spin correlation functions were decoupled when $T>T_{c}$. The region of validity of this decoupling scheme and the motivation for its use will be examined shortly in connection with a procedure we have developed in which the positional correlation of the spins is considered in detail. From this calculation the importance of short range order and its effect on the spectral density when $T \gtrsim T_{c}$ will become quite apparent. 

frequency distribution of the neutron scattering cross section is contained in $G^{\alpha \beta}(\vec{q}, w)$.

In order to simplify the following calculation a system will be considered in which the spins are localized at the ionic sites of the lattice, and so

$$
\vec{S}(\vec{r})=\sum_{i} \vec{S}_{i} \delta\left(\vec{r}-\vec{R}_{i}\right)
$$

Then

$$
\overrightarrow{\vec{s}}_{\vec{q}}=\frac{1}{\sqrt{N}_{N}} \sum_{i} \vec{s}_{i} e^{i \vec{q} \cdot \vec{R}_{i}},
$$

where the sum on $i$ is carried over all $N$ lattice vectors $\vec{R}_{i}$. For this localized spin system, $G^{O B}(\vec{q}, w)$ can be written in the following way:

$$
G^{\alpha \beta}(\vec{q}, \omega)=\frac{1}{2 \pi} \int_{-\infty}^{\infty}\left\langle s_{\vec{q}}^{\alpha}(0) s_{-\vec{q}}^{\beta}(t)\right\rangle e^{-i \omega t} d t .
$$

When this expression is integrated over all frequencies we obtain

$$
\begin{aligned}
\int_{-\infty}^{\infty} G^{\alpha \beta}(\vec{q}, \omega) d \omega & =\frac{1}{2 \pi} \int_{-\infty}^{\infty} d \omega \int_{-\infty}^{\infty}\left\langle s_{\vec{q}}^{\alpha}(0) s^{\beta}(t)\right\rangle e^{-i \omega t} d t \\
& =\int_{-\infty}^{\infty}\left\langle s^{\alpha}(0) s^{\beta}(t)\right\rangle \delta(t) d t \\
& =\left\langle s_{\vec{q}}^{\alpha} s^{\beta}\right\rangle,
\end{aligned}
$$

which is just the equilibrium spin polr. correlation function. For a system with isotropic interactions the neutron scattering cross section, Eq. $(111.1)$, reduces to 
$\frac{d^{2} \sigma}{d \Omega^{\prime} d E^{\prime}} \propto \sum_{\alpha}\left[1+\left(\hat{q}^{\alpha}\right)^{2}\right] G^{\alpha \alpha}(\vec{q}, \omega)$,

where $\alpha=x, y, z$.

In the paramagnetic phase

$\left\langle\underset{\vec{q}}{s^{x}}(0) s_{-\vec{q}}^{x}(t)\right\rangle=\left\langle\underset{\vec{q}}{s^{y}}(0) s_{-q}^{y}(t)\right\rangle=\left\langle\underset{\vec{q}}{\left\langle s^{z}\right.}(0) s_{-\vec{q}}^{z}(t)\right\rangle$

since there is no overall preferred direction of spin orientation. This implies that

$$
G^{X X}(\vec{q}, w)=G^{Y y}(\vec{q}, w) \equiv G^{Z Z}(\vec{q}, w) \equiv G(\vec{q}, w) .
$$

Consequently, above $T_{c}$

$$
\frac{d^{2} \dot{\sigma}}{d \Omega^{\prime} d E^{\prime}} \propto G(\vec{q}, \omega) \text {. }
$$

Therefore, since the neutron scattering cross section is directly proportional to $G(\vec{q}, \omega)$ in this temperature region, a comparison between $G(\vec{q}, \omega)$ and the neutron cross section determined from the measured scattering intensity can be readily accomplished. However, before we consider the properties of this function in more detail, its relation to the spin relaxation function and spectral density function which have been used in prior theoretical work (see Chapter 11) will be examined.

In studying the behavior of spontaneous spin fluctuations, Marshall and Lowde 44 have examined the properties of the spin relaxation function 


$$
R^{\alpha \beta}(\vec{q}, t)=\left\{\underset{-\vec{q}}{\alpha s^{\alpha}}(0), \underset{\vec{q}}{s^{\beta}}(t)\right\}
$$

which describes how a spin system relaxes after a disturbance is removed. The Fourier transform of $R^{\infty \beta}(\vec{q}, t)$ is

$$
r^{\infty \beta}(\vec{q}, \omega)=\frac{1}{2 \pi} \int_{-\infty}^{\infty} e^{-i \omega t} R^{\alpha \beta}(\vec{q}, t) d t
$$

When $T>T_{c}$, then by definition

$$
\left.\underset{-\vec{q},}{\left\{s^{\alpha}\right.}(0), s_{\vec{q}}^{\beta}(t)\right\}=\int_{0}^{\beta} d \lambda\left\langle e^{\lambda H} s_{-\vec{q}}^{\alpha}(0) e^{-\lambda H} s_{\vec{q}}^{\beta}(t)\right\rangle
$$

and

$$
r^{\alpha \beta}(\vec{q}, \omega)=\frac{1}{2 \pi} \int_{-\infty}^{\infty} d t e^{-i \omega t} \int_{0}^{\beta} d \lambda\left\langle e^{\lambda H} s_{-\vec{q}}^{\alpha}(0) e^{-\lambda H} e^{i H t} s_{\vec{q}}^{\beta}(0) e^{-i H t}\right\rangle .
$$

The refore,

$$
\begin{aligned}
r^{\alpha \beta}(\vec{q}, w)= & \frac{1}{2} \sum_{i]} \int_{-\infty}^{\infty} d t e^{-i\left[\omega-\left(E_{j}-E_{i}\right)\right] t} \\
& x \int_{0}^{\beta} d \lambda z^{-1} e^{-\beta E_{i}-\lambda\left(E_{j}-E_{i}\right)} \\
& x\left\langle i\left|s_{-\vec{q}}^{\alpha}(0)\right| j\right\rangle\left\langle j\left|s_{\vec{q}}^{\beta}(0)\right| i\right\rangle,
\end{aligned}
$$

where

$$
s_{\vec{q}}^{\alpha}(t)=e^{i H t} s_{\vec{q}}^{\alpha}(0) e^{-i H t}
$$


$|i\rangle$ and $|j\rangle$ are eigenstates of the spin Hamiltonian $H$ with energies $E_{i}$ and $E_{j}$ respectively, and $z$ is the partition function. Then

$$
\begin{aligned}
r^{\alpha \beta}(\vec{q}, \omega)= & \sum_{i j} \delta\left[\omega-\left(E_{j}-E_{i}\right)\right] z^{-1} e^{-\beta E_{i}} \\
& \left\langle i\left|s_{-\vec{q}}^{\alpha}(0)\right| j\right\rangle\left\langle j\left|s_{\vec{q}}^{\beta}(0)\right| i\right\rangle \int_{0}^{\beta} e^{-\lambda \omega} d \lambda, \\
= & \frac{\left(1-e^{-\beta \omega}\right)}{\omega} \frac{1}{2 \pi N} \int_{-\infty}^{\infty} e^{-i \omega t} z^{-1} \\
& x \sum_{i j} e^{-\beta E}\left\langle i\left|s_{-\vec{q}}^{\alpha}(0)\right| j\right\rangle\left\langle j\left|e^{i H t} s_{\vec{q}}^{\beta}(0) e^{-i H t}\right| i\right\rangle, \\
\because \quad & N \frac{\left(1-e^{-\beta \omega}\right)}{\omega} \int_{-\infty}^{\infty} e^{-i \omega t}\left\langle s^{\alpha}(0) s_{\vec{q}}^{\beta}(t)\right\rangle,
\end{aligned}
$$

so :

$$
G^{\alpha p}(\vec{q}, w)=\frac{N^{-1} w}{\left(1-e^{-\beta \omega}\right)} r^{a p}(\vec{q}, w)
$$

In the above calculation $\hbar=1$ and the factor $\omega /\left(1-\mathrm{e}^{-\beta \omega}\right)$ takes into arcount the detailed balance between the magnon absorption and emission processes that occur when neutrons are scattered inelastically by the spin system.

The relation between $G^{\alpha \beta}(\vec{q}, w)$ and the dynamical susceptibility can be derived in the following way. In a straight forward proof it can be shown

$$
\frac{\partial}{\partial t}\{A, B(t)\}=i\langle[A, B(t)]\rangle \text {. }
$$


Consequently,

$$
\begin{aligned}
\frac{\partial}{\partial t} R^{\alpha \beta}(\vec{q}, t) & =-\left\langle\left[s_{-\vec{q}}^{\alpha}(0), s_{\vec{q}}^{\beta}(t)\right]\right\rangle \\
& =-\frac{N}{g^{2} \mu^{2}} x^{\alpha \beta}(\vec{q}, t),
\end{aligned}
$$

where $x^{\propto B}(\vec{q}, t)$ is the dynamical response function which determines the response of the $\vec{q}^{\text {th }}$ wavevector of the magnetization to an applied field $\vec{H}(\vec{q}, t)$ :

$$
M^{\alpha}(\vec{q}, t)=\int d t^{\prime} x^{\alpha \beta}\left(\vec{q}, t-t^{\prime}\right) H^{\beta}\left(\vec{q}, t^{\prime}\right)
$$

in the case where the system is translationally invariant and stationary. Since the principle of causality imposes the following restriction on the response function:

$$
x^{\infty}(\vec{q}, t)=0
$$

when $t<0$, then we can define the generalized susceptibility to be 30

$$
x^{\alpha \beta}(\vec{q}, \omega)=\int_{0}^{\infty} e^{-i \omega t} x^{\alpha \beta}(\vec{q}, t) e^{-\epsilon t} d t
$$

where $\epsilon \rightarrow 0^{+}$. The presence of $e^{-\epsilon t}$ insures that the response is adiabatic.

Using this definition and Eq. (111..18) we obtain

$$
x^{\alpha \beta}(\vec{q}, \omega)=\frac{-g^{2} \mu^{2}}{N} \int_{0}^{\infty} e^{-i \omega t} \frac{\partial}{\partial t} R^{\alpha \beta}(\vec{q}, t) d t,
$$


y

which when integrated by parts becomes

$$
\therefore x^{\alpha \beta}(\vec{q}, \omega)=\frac{q^{2} \mu^{2}}{N} R^{\alpha \beta}(\vec{q}, 0)-\frac{q^{2} \mu^{2}}{N} i \omega \int_{0}^{\infty} e^{-i \omega t} R^{\alpha \beta}(\vec{q}, t) d t .
$$

Since the canonical correlation function has the property

$$
\{A, B\}=\{B, A\}
$$

then

$$
R^{\alpha \beta}(\vec{q}, t)=R^{\beta \alpha}(-\vec{q},-t)=R^{\alpha \beta}(-\vec{q}, t) *
$$

This result along with Eq. (III.24) implies that

$$
x^{\alpha \beta}(\vec{q}, w)=x^{\alpha \beta}(-\vec{q},-w)^{*}
$$

Consequently,

$$
\begin{aligned}
x^{\alpha \beta}(\vec{q}, \omega)-x^{\beta \alpha}(\vec{q}, \omega)^{*} & =-\frac{g \mu^{2}}{N} i \omega \int_{-\infty}^{\infty} e^{-i \omega t} R(\vec{q}, t) d t \\
& =\frac{-g^{2} \mu^{2}}{N} 2 \pi i \omega r^{\alpha \beta}(\vec{q}, \omega) .
\end{aligned}
$$

Now when $\alpha=\beta$, the above equation becomes

$$
\frac{x^{\alpha+1}(\vec{q}, \omega)}{\omega}=\frac{g^{2} \mu^{2}}{N} 2 \operatorname{Re}\left[r^{\alpha \alpha}(\vec{q}, \omega)\right]
$$

where $x^{\infty \alpha^{\prime}}$ is the imaginary component of the generalized susceptibility which in this case governs the dissipation of energy caused by the excitation of spin fluctuations in the system. Then using Eq. (111.17) 
we obtain

$$
\frac{x^{\infty}{ }^{\prime \prime}(\vec{q}, \omega)}{\omega}=\frac{\left(1-e^{\left.-\beta_{\omega}\right)}\right.}{\beta_{\omega}} G^{\alpha \alpha}(\vec{q}, \omega)
$$

which is simply a statement of the fluctuation-dissipation theorem. Here the term on the left hand side is usually referred to as the spectral density. Thus because of the above interrelationships between $G^{\infty \beta}(\vec{q}, w)$, the relaxation function, the spectral density, and the generalized susceptibility, it is desirable to obtain an explicit expression for $G^{\alpha \beta}(\vec{q}, \omega)$.

Below the critical temperature the spin system exhibits long range order, and as $T \rightarrow 0^{\circ} \mathrm{K}$ the spins take on the $\mathrm{Ir}$ maximum allowable quantum number along the axis of quantization, namely $S$. Consequently, at temperatures near absolute zero spin wave theory may be used to evaluate the spin pair correlation functions, and thus in this. temperature region we find that 44

$$
G^{x x}(\vec{q}, w) \cdot \alpha \frac{z}{2}\{\delta[w-w(\vec{q})]+S[w+w(\vec{q})]\},
$$

where $\hbar_{w}(\vec{q})$ is the energy of a noninteracting spin wave mode of wave vector $\vec{q}$ :

$$
\hbar_{\omega}(\vec{q})=2 \hbar S[J(0)-J(\vec{q})]
$$

Here $J(\vec{q})$ is the Fourier transform of the exchange interaction. So at low temperatures $G^{x X}(\vec{q}, w)$ is expressed in terms of delta function singularities which occur at frequencies corresponding to the absorption 
or emission of a spin wave mode excited by the inelastic scattering of neutrons. As the temperature increases, spin wave interactions become important and act to give a finite line width to the peaks in $G^{x \times}(\vec{q}, w)$. However at temperatures near the critical temperature an exact form for $G^{Q \alpha}(\vec{q}, w)$ is impossible to obtain.

In order to clearly ascertain what difficulties are involved in the calculation $G^{\alpha \alpha}(\vec{q}, \omega)$ near $T_{c}\left(T \gtrsim T_{c}\right)$, we will now construct a serles expansion for the dynamical spin correlation function

$$
g^{\alpha \alpha}(\vec{q}, t)=\left\langle s_{\vec{q}}^{\alpha}(0) s_{-\vec{q}}^{\alpha}(t)\right\rangle
$$

and use the result to obtain $G^{\alpha \alpha}(\vec{q}, \omega)$, since

$$
G^{\infty \alpha}(\vec{q}, \omega)=\frac{1}{2 \pi} \cdot \int_{-\infty}^{\infty} g^{\infty \alpha}(\vec{q}, t) e^{-i \omega t} d t
$$

We begin by expanding $g^{\alpha \alpha}(\vec{q}, t)$ into a Taylor series in powers of $t$.

$$
g^{\infty \alpha}(\vec{q}, t) \stackrel{\sum}{=} \frac{(-i t)^{n}}{n !}\langle G \underset{\vec{q}}{(n)}\rangle, \infty \alpha
$$

whe re

$$
\langle G \underset{\vec{q}}{(n)}\rangle^{\infty \alpha}=\left[\left(\frac{1}{i} \frac{\partial}{\partial t}\right)^{n}\left\langle\underset{\vec{q}}{s^{\alpha}}(0) \underset{-\vec{q}}{s^{\alpha}}(t)\right\rangle\right]_{t=0}
$$

As a consequence of the fact that $s_{-\vec{q}}^{\alpha}(t)$ is a Heisenberg operator, i.e.

$$
s_{-\vec{q}}^{\alpha}(t)=e^{i H t} s_{-\vec{q}}^{\alpha}(t) e^{-i H t}
$$


when $H$ is time independent, and

$$
\dot{s}_{-\vec{q}}^{i \alpha}(t)=i\left[H, s_{-\vec{q}}^{\alpha}(t)\right]
$$

then the coefficients in the above series expansion can be expressed in terms of multiple commutators of the Heisenberg spin Hamiltonian

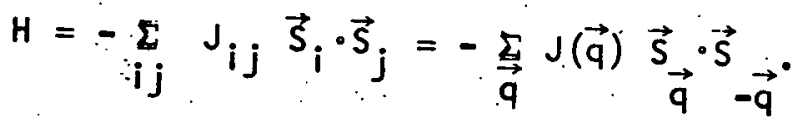

So through the use of Eq. $(111.38)$ and cyclic property of the trace it can be readily shown that

$$
\begin{aligned}
& \left\langle G_{\vec{q}}^{(0)}\right\rangle^{\alpha \alpha}=\left\langle s_{\vec{q}}^{\alpha} s_{-\vec{q}^{\alpha}}\right\rangle, \\
& \left\langle G_{\vec{q}}^{(1)}\right\rangle^{\alpha \alpha}=\left\langle s_{\vec{q}}^{\alpha}\left[H, s_{-\vec{q}}^{\alpha}\right]\right\rangle, \\
& \left\langle G_{\vec{q}}^{(2)}\right\rangle^{\alpha \alpha}=\left\langle\left[_{\vec{q}^{\alpha}}^{\alpha}, H\right]\left[H, s_{-\vec{q}}^{\alpha}\right]\right\rangle,
\end{aligned}
$$

etc. Since each commutation with $H$ brings in another pair of spins, it is evident that the coefficients $\langle\underset{G}{(n)})^{\infty}$ in the series expansion of the time-dependent spin correlation function are equal to progressively higher order static multi-spin correlation functions.

The problem of calculating the time dependent spin correlation function now becomes clear when Eq. (111.35) is examined: at temperatures near $T_{c}$ critical spin fluctuations bexome very important and corsequently an expression for $G^{\infty \alpha}(\vec{q}, w)$ must be obtained which is valid not only for 

In the random phase approximation $\nu=\frac{1}{2}$. However, in more exact theories $\nu \cong \frac{2}{3}$, within the critical region where $k \lambda \gg 1$ and where both $k^{-1}$ and $\lambda$ are macroscopic lengths. However, since we will be examining the behavior of spin fluctuations outside the critical region where $k \lambda \gtrsim 1, \nu=\frac{1}{2}$ can be used.

The experimental determination of the correlation length follows directly from the definition of the neutron scattering cross section where, from Eqs. (111.1), (111.2) and (111.5),

$$
\frac{d^{2} \sigma}{d \Omega^{2} d \omega^{1}} \propto \frac{1}{2 \pi} \int_{-\infty}^{\infty} e^{-i \omega t}\left\langle s_{\vec{q}}^{\alpha}(0) s_{-q}^{\alpha}(t)\right\rangle d t
$$

then integrating over all energies of the scattered neutrons we obtain

$$
\int \frac{d^{2} \sigma}{d \Omega^{\prime} d \omega^{\prime}} d \omega \propto \frac{1}{2 \pi} \int d \omega \int d t e^{-i \omega t}\left\langle s_{\vec{q}}^{\alpha}(0) s_{-\vec{q}}^{\alpha}(t)\right\rangle \propto\left\langle s_{\vec{q}}^{\alpha} s_{-\vec{q}}^{\alpha}\right\rangle_{0}
$$

Consequently, for small q

$$
\int \frac{d^{2} \sigma}{d \Omega^{\prime} d \omega^{\prime}} d \omega^{\prime} \propto \frac{1}{q^{2}+(i / \lambda)^{2}}
$$

Thus $\lambda$ may be obtained by fitting the expression on the left hand side of $\mathrm{Eq}$. (111.47) to the experimentally determined total integrated scattering intensity. 
IV. CALCULATION OF THE LOWEST ORDER COEFFICIENTS IN THE SERIES EXPANSION FOR THE TRANSVERSE AND LONGITUDINAL

\section{CORRELATION FUNCTIONS}

A. Importance of Short Range Order above the Critical Temperature For the present study the isotropic Heisenberg model will be examined:

$$
H=-\sum_{i j} J_{i j} \vec{s}_{i} \cdot \vec{s}_{j}=-\sum_{\vec{q}} J(\overrightarrow{\vec{a}}) \vec{s}_{\vec{q}}^{\vec{s}}-\vec{q},
$$

where

$$
J(\vec{q})=\sum_{j} J_{i j} e^{-i \vec{q} \cdot \vec{R}} \mathbf{i j}
$$

and

$$
\vec{\xi}_{\vec{q}}=\frac{l}{\sqrt{N} \sum_{j}} \vec{s}_{j} e^{-i \vec{q} \cdot R_{j}}
$$

Here $J_{i j} g i v e s$ the exchange interaction between the $i^{\text {th }}$ and $j^{\text {th }}$ spins $J_{i j}=J\left(\vec{R}_{i}-\vec{R}_{j}\right)$. In practice these exchange parameters are considered to be phenomenological constants whose values are determined by fitting this model to the experimental data.

The motivation for our consideration of this particular system comes from the fact that it is inherently much simpler to treat than the itinerant electron theory of ferromagnetism and from the fact that one observes magnon-like excitations above the critical temperature in 
neutron scattering experiments not only in metals such as nickel 17 and iron $^{46}$ but also in magnetic insulators like RbMnF $_{3}{ }^{18}$

From Eq. $(111.10)$ it is evident that in order to determine the shape of the inelastic neutron scattering cross section due to spin fluctuations above the critical point the function $G^{\alpha \alpha}(\vec{q}, \omega)$ must be calculated. This calculation will. now be accomplished through the use of the series expansion of the dynamical spin correlation function in powers of t, Eq. (111.35). The usefulness of this approach lles in the fact that the coefficients, $\langle\underset{\vec{q}}{(n)}\rangle^{c \alpha}$, in this power series can be related to static multiple spin correlation functions. We can readily evaluate the lowest order coefficients by employing a physical approximation which is developed in this section. Because of the resulting simple expressions for the coefficients of the zeroth through second power of $t$, we will incorporate them as leading terms in a resummation which is used to determine an approximate expression for the dynamical spin correlation function, Eq. $(111.33)$, from which $G^{\alpha O \alpha}(\vec{q}, \omega)$ can be found by Fourier transformation.

We now proceed with a calculation of $G^{\alpha \alpha}(\vec{q}, w)$ above the critical temperature. In Chapter 111 we define

$$
G^{\alpha \alpha}(\vec{q}, \omega)=\int_{-\infty}^{\infty} g^{\alpha \alpha}(\vec{q}, t) e^{-i \omega t} d t
$$

where $\alpha=x, y, z$ and

$$
g^{\alpha \alpha}(\vec{q}, t)=\left\langle s_{\vec{q}}^{\alpha}(0) s_{-q}^{\alpha}(t)\right\rangle .
$$


Then using the expansion of $g^{\alpha \alpha}(\vec{q}, t)$ in powers of $t$, we obtain

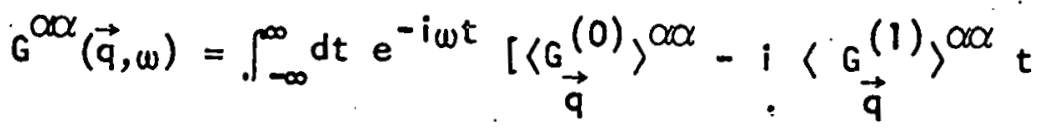

$$
\begin{aligned}
& \left.-\frac{1}{2 !}\langle\underset{\vec{q}}{(2)}\rangle^{\alpha O t^{2}}+\frac{i}{3 !}\left\langle\underset{\vec{q}}{\left\langle G^{(3)}\right.}\right\rangle^{O O t^{3}}+\cdot \cdot \cdot\right]
\end{aligned}
$$

The multiple commutator expressions for the lowest order coefficients in the above series are given by Eqs. $(111.40)-(11-1.42)$ and

$$
\left.\left\langle G_{\vec{q}}^{(3)}\right\rangle^{\alpha \alpha}=\left\langle S_{\vec{q}}^{\alpha}, H\right]\left[H,\left[H, s_{-\vec{q}}^{\alpha}\right]\right]\right\rangle,
$$

where $H$ is the Heisenberg exchange Hamiltonian. It will be very apparent from the complete calculation of $\left\langle G_{\vec{G}}(3), 00 x\right.$ (Appendix A) that an explicit derivation of any of the higher order coefficients would be extremely tedious, and consequently we will carry out detailed calculations for only $\langle\underset{\vec{q}}{(0)}\rangle$ through $\langle\underset{\vec{q}}{(3)}\rangle$.

Our calculation of these coefficients can be simplified by making the following observation. When $T \gtrsim T, c$, and the neutron scattering wave vector satisfies the inequality $q>\lambda^{-1}$ where $\lambda$ is the spin pair correlation. length discussed in Chapter 111 , then from the theory of Fourier transformations the dominant contribution to the spatial sum in the equation for the Fourier transform of the dynamical spin correlation function, 

order coefficients in the series expansion of the spin correlation function,

$$
\begin{aligned}
& g^{\infty \alpha}(\vec{q}, t)=\sum_{j}\left(\left\langle G_{i j}^{(0)}\right\rangle^{\infty \alpha}-i\left\langle G_{i j}^{(i)}\right\rangle^{\infty \alpha} t\right. \\
& \left.-\frac{1}{2}\left\langle G_{i j}^{(2)}\right\rangle^{\alpha \alpha} t^{2}+\cdots \cdot\right) e^{-i \vec{q} \cdot \vec{R}_{i j}} \text {, }
\end{aligned}
$$

where

$$
\left\langle G_{i j}^{(n)}\right\rangle^{\infty \alpha}=\left[\left(\frac{1}{i} \frac{\partial}{\partial t}\right)^{n}\left\langle s_{i}^{\alpha}(0) s_{j}^{\alpha}(t)\right\rangle\right]_{t=0}
$$

comes from spins which lie within regions whose sizes are measured by $\lambda$. The reason for this is that each succeeding coefficient involves a higher order time derivative of the dynamical correlation function which introduces a multiple commutator of the Heisenberg Hamiltonian $H$ with the spin operator. Each commutation of $H$ with $s_{j}^{\alpha}$ brings in the appropriate components of a set of spins at sites $l$ which are coupled to $s_{j}^{\alpha}$ via the exchange interaction $J_{j l}$. Consequently, when $r<q^{-1}<\lambda$, all of the spins at sites $\ell$ lie within a correlated spin cluster. Thus when the above inequality, Eq. (iv, II) holds, the major contribution to the dynamical spin correlation function $g^{\infty \alpha}(\vec{q}, t)$ above the critical temperature is due to clusters of correlated spins. Hence it is evident that positional spin correlations are very important near the critical temperature and must be explicitly accounted for. In order to do this we must introduce a local order parameter which is a measure of the 
degree of spin order within a correlated cluster. McLean and Blume 42 first introduced the concept of a local order parameter so that they could account for the existence of short range order in one dimensional Heisenberg magnets. This parameter was oriented in the direction of the local magnetization. The underlying assumption of their theory is that the spatial and temporal variations of the parameter occur slowly near $T=0$ so that in the first approximation the local order parameter is constant with respect to the propagation of short wavelength $\left(q \gg \lambda^{-1}\right)$ spin waves.

This definition will be followed rather closely here. However, in order to simplify our calculations, we will construct a time independent local order parameter. Since the normalized correlation function $\left\langle\vec{s}_{i} \cdot \vec{s}_{j}\right\rangle / s(s+1)$ gives the probability that spins on sites $i$ and $j$ are correlated. with each other and thus is a measure of the spin alignment, we will take this to be our local order parameter. This parameter also has a direction which is that of the local magnetization associated with the spin cluster. We define this to be the direction of the local $z$ axis. .

Consequently, in discussing the contributions to the lowest order coefficients in the power series expansion of the dynamical spin correlation function; we explicitly account for the existence of short range order above the critical temperature by approximating the system as a collection of correlated. spin clusters whose orientations are distributed randomly and whose degree of spin alignment is given by the local order parameter. 
When the system is approximated in this way, the time dependent spin pair correlation function $\left\langle s_{i}^{\alpha}(0) s_{j}^{\alpha}(t)\right\rangle$ can be written in the $f$ following way:

$$
\begin{aligned}
\left\langle s_{i}^{\alpha}(0) s_{j}^{\alpha}(t)\right\rangle= & \frac{1}{3}\left[\left\langles_{i}^{z}(0) s_{j}^{z}(t)+\frac{1}{2}\left\langle\left\langle s_{i}^{+}(0) s_{j}^{-}(t)\right\rangle+\right.\right.\right. \\
& \left.\left.\left\langle s_{i}^{-}(0) s_{j}^{+}(t)\right)\right)\right],
\end{aligned}
$$

where

$$
s_{j}^{ \pm}=s_{j}^{x} \pm i s_{j}^{y}
$$

Here these spin operators are defined with respect to: the local $z$ axis of the spin cluster which is the axis of quantization. The factor of $\frac{1}{3}$ takes into account the fact that the local $z$ axis is randomily oriented in the spin system under consideration.

As a consequence of Eq. (IV.4), we can write

$$
G^{\alpha \alpha}(\vec{q}, w)=\frac{1}{3} G^{z z}(\vec{q}, w)+\frac{1}{6}\left[G^{+-}(\vec{q}, w)+G^{+}(\vec{q}, w)\right],
$$

where we can define

$$
G^{T}(\vec{q}, w)=\frac{1}{2}\left[G^{+-}(\vec{q}, w)+G^{-+}(\vec{q}, \dot{w})\right]
$$

Then Eq. (IV.17) has the same form as that derived by McLean and BI ume. 42 It should be noted however that it is valid only for $T>T_{c}$ when the system is approximated in the above discussed manner. Thus one of the most significant features of the inclusion of local order above the ritical point is that instead of a single correlation function both 
the transverse and longitudinal correlations with respect to the axis of quantization, the local $z$ axis, must be considered.

In Section B we will calculate the first and second order coefficients of the powers of $t$ in the series expansion for the transverse dynamical spin correlation function $g^{\top}(\vec{q}, t)$ where

$$
g^{\top}(\vec{q}, t)=\frac{1}{2}\left[g^{+-}(\vec{q}, t)+g^{+}(\vec{q}, t)\right] .
$$

An approximate expression for $g^{\top}(\vec{q}, t)$ will then be obtained by a resummation and $G^{\top}(\vec{q}, w)$ obtained by Fourier transformation. In Section $C$ an equation for $G^{2 Z}(\vec{q}, \omega)$ will be determined.

\section{Determination of $G^{\top}(\vec{q}, w)$}

Since $G^{+-}(\vec{q}, w)$ and $G^{-+}(\vec{q}, w)$ may be calculated in a parallel manner, we will concentrate on the evaluation of $G^{+-}(\vec{q}, \omega)$. From this discussion the expression for $G^{-+}(\vec{q}, w)$ will follow directly.

As discussed in Chapter 111 , Eg. $(111.35)$, the dynamical spin correlation function can be expanded in a Taylor series in powers of $t$. Thus

$$
g^{+}(\vec{q}, t)=\sum_{n=0}^{\infty} \frac{(-1 t)^{n}}{n !}\langle\underset{\vec{q}}{(n)}\rangle^{+-},
$$

and so

$$
\begin{gathered}
G^{+-}(\vec{q}, \omega)=\int_{-\infty}^{\infty} d t e^{-i \omega t}\left[\left\langle G_{\vec{q}}^{(0)}\right\rangle^{+-}-i\left\langle G_{\vec{q}}^{(1)}\right\rangle_{t}\right. \\
-\frac{1}{2 !}\left\langle G_{\vec{q}}^{(2)}\right\rangle t^{2}-\cdots \cdot
\end{gathered}
$$




\section{6}

From Eqs. (111.40 and 111.42 ) we have the result that

$$
\begin{aligned}
\left\langle G_{\vec{q}}^{(0)}\right\rangle^{+-} & =\left\langle\underset{\vec{q} \cdot s_{-\vec{q}}^{+}}{s^{-}}\right\rangle, \\
\left\langle G_{\vec{q}}^{(1)}\right\rangle^{+-} & =\left\langle\underset{\vec{q}}{\left\langle s^{+}\right.}\left[H_{-\vec{q}}^{-}\right]\right\rangle
\end{aligned}
$$

and

$$
\langle\underset{\vec{q}}{\langle(2)}\rangle^{+-}=\left\langle\left[S_{\vec{q}}^{+}, H\right]\left[H, S_{-q}^{-}\right]\right\rangle
$$

Now in order to determine exactly how the presence of positional correlations of the spins above $T_{c}$ affects the calculation of the lowest order coefficients, the spatial fourier transform of the first order coefficient will be examined:

$$
\langle\vec{q}(1)\rangle^{+-}=\sum_{j}\langle G(1)\rangle^{(--} e^{i \vec{q} \cdot \vec{R}_{i j}},
$$

where

$$
\left\langle G_{i j}^{(1)}\right\rangle^{+-}=\left\langle s_{i}^{+}\left[H, s_{j}^{-}\right]\right\rangle .
$$

Here Eqs. (IV.1-IV.3) have been used in the derivation of these expressions.

Using Eq. (IV.1) and the following commutator relations

$$
\begin{aligned}
& {\left[s_{i}^{2}, s_{j}^{+}\right]=\delta_{i j} s_{i}^{+},} \\
& {\left[s_{i}^{2}, s_{j}^{-}\right]=\delta_{i j} s_{i}^{-}}
\end{aligned}
$$



where

$$
\begin{aligned}
& A_{i j}^{+-}(1)=\left\langle S_{i}^{+}\left[H, S_{j}^{-}\right]\right\rangle_{s w} P, \\
& B_{i j}^{+-}(1)=\left\langle S_{i}^{+}\left[H, S_{j}^{-}\right]\right\rangle(1-P)
\end{aligned}
$$

in which the significance of $\langle. . .\rangle_{\text {sw }}$ is described directly below.

An expression for $P$ may be determined in the following way. Since we have assumed that the range of the exchange interaction is much less than the two spin correlation length $\lambda$, then if the spins at sites $i$ and $\mathbf{j}$ are within a distance $\lambda$ of each other, all the spins at sites $i, j$, and $\ell$ will lie within this region. So the probability $P$ may simply be equated to the normalized two-spin correlation function

$$
p \equiv \rho_{i j}=\frac{\left\langle\vec{s}_{i} \cdot \vec{s}_{i}\right\rangle}{s(s+1)} \text {. }
$$

When the spins on sites $i$ and $j$ are aligned, $\rho_{i j}=1$; and when they are statistically independent, $\rho_{i j}=0$. Thus $p$ is the magnitude of the local order parameter in the spatial region near the pair of spins $i$ and $\mathbf{j}$

In evaluating $A_{i j}^{+-}(1)$, wo note that all the spins contained in the multi-spin correlation functions comprising $A_{i j}^{+-}(1)$ fall withincorrelated spin clusters. Therefore, these correlation functions will be approximated through the use of spin wave theory for an infinite crystal, which is symbolized by $\langle. . .\rangle_{s w}$. This is done by letting

$$
s_{i}^{+} \simeq \sqrt{2 S} a_{i}
$$




$$
s_{i} \simeq \sqrt{2}_{2} a_{i}
$$

and

$$
s_{i}^{z}=-s+a_{i}^{\dagger} a_{i}
$$

whe re

$$
\begin{aligned}
& a_{i}=\frac{1}{\sqrt{N}} \underset{k}{\sum_{k}} a^{i \vec{k} \cdot \vec{k}_{j}}, \\
& a_{i}^{+}=\frac{1}{\sqrt{N}} \sum_{\vec{k}} \vec{a}_{\vec{k}}^{\dagger} e^{-\vec{k} \cdot \vec{R}_{i}},
\end{aligned}
$$

in which $a_{\vec{k}}^{+}$and $a_{\vec{k}}$ are the magnon creation and annihilation operators respectively. The above expressions are just the Holstein-Primakoff spin operator expansions" in which only the lowest order terms retained. Then

$$
\left\langle s_{i}^{+} s_{l}^{-} s_{j}^{z}\right\rangle \simeq s(2 s)\left\langle a_{i} a_{l}^{+}\right\rangle
$$

and $A_{i j}^{+-}(1)$ has the form

$$
A_{i j}^{+-}(1)=-2 S \sum_{l} J_{j l}\left\{2 S\left[\left\langle a_{i} a_{l}^{\dagger}\right\rangle-\left\langle a_{i} a_{j}^{\dagger}\right\rangle\right]\right\}_{i j},
$$

where

$$
\left\langle a_{i} a_{l}^{+}\right\rangle=\frac{1}{N} \sum_{\vec{k}}\left(i+\left\langle n_{\vec{k}}\right\rangle\right) e^{i \vec{k}, \vec{R}_{i l}},
$$

since $\left[\vec{k}_{\vec{k}^{\prime}}^{a^{+} \vec{k}^{\prime}}\right]=\vec{k}_{\vec{k}, \vec{k}^{\prime}}$ and ${ }_{\vec{k}}=\underset{\vec{k}}{a^{\dagger}} \vec{k}$ which is the magnon number operator. 
It should be noted that by using this approximation we assume that the spins within the spin cluster tend to be aligned along the local z axis of the cluster.

We will now evaluate the multiple spin correlation functions in $B_{i j}^{+\infty}(1)$. Since the spins in this term lie outside the spin clusters the multiple spin correlation functions may be approximated through the use of the cumulant expansion method employed by Reiter; 40

$$
\left\langle s_{i}^{+} s_{l}^{-} s_{j}^{z}\right\rangle \simeq\left\langle s_{i}^{+} s_{l}^{-}\right\rangle\left\langle s_{j}^{z}\right\rangle,
$$

where $\langle. .$.$\rangle is an average over an equilibrium canonical ensemble.$ However, $\left\langle S_{j}^{z}\right\rangle=0$ when $T>T_{c}$, since there is no perferred direction for spins lying outside the spin clusters. Consequently,

$$
B_{i j}^{+\infty}(1)=0
$$

The above argument can be extended to include all odd ordered coefficients so that

$$
\mathrm{B}_{1 \mathrm{j}}^{2-}(2 n+1)=0 ; n=0,1,2, \ldots
$$

This is a result of the fact that the multi-spin correlation functions comprising $B_{i j}^{+-}(2 n+1)$ must be rotationally invariant because the collection of spins lying outside the spin clusters have this symmetry property. But the correlation functions in $B_{i j}^{+-}(2 n+1)$ contain odd numbers of spins, and thus the requirement of rotational invariance fnrces these equilibrium ensemble averages to be identically equal to - 'ro. 
As a consequence of this result $\left\langle G_{\vec{q}}^{(1)}\right\rangle^{+-}$is determined solely by. the spin cluster contribution, $A_{i j}^{+-}(1)$. We can obtain an explicit expression for the first order coefficient by taking the Fourier transform of $A_{i j}^{+-}(1)$. Since

$$
\rho_{i j}=\frac{1}{N} \sum_{\vec{q}} \rho(\vec{q}) e^{i \vec{q} \cdot \vec{R}} i j,
$$

where

$$
\because\left\langle s_{i}^{z} s_{j}^{z}\right\rangle=\frac{2}{2}\left\langle s_{i}^{+} s_{j}^{-}\right\rangle=\frac{1}{3} s(s+1) p_{i j},
$$

then for $T \gtrsim T_{c}$ and $r<q^{-1}<\lambda$

$$
\left.\underset{\vec{q}}{\left\langle G^{(1)}\right.}\right\rangle^{+-}=\frac{2 S}{N} \underset{\vec{q}^{\prime}}{\sum} \omega\left(\vec{q}^{\prime}\right)\left[\left\langle n_{\vec{q}^{\prime}}\right\rangle+1\right] \rho\left(\vec{q}-\vec{q}^{\prime}\right),
$$

with

$$
\left\langle\vec{q}_{\vec{q}^{\prime}}\right\rangle=\frac{i}{e^{\beta \omega\left(q^{\prime}\right)-1}},
$$

and

$$
\omega(\vec{q} !)=2 S\left[J(0)-J\left(\vec{q}^{\prime}\right)\right] .
$$

The calculation of the second order coefficient $\underset{\vec{q}}{\left\langle G^{(2)}\right\rangle^{+-}}$is done in an analogous manner. Since

$$
\left.\underset{\vec{q}}{\left\langle G^{(2)}\right.}\right\rangle^{+-}=\sum_{j}\left\langle G_{i j}^{(2)}\right\rangle^{+-} e^{-i \vec{q} \cdot \vec{R}_{i j}},
$$

then when $r<q^{-1}<\lambda$ the dominant contribution comes from those terms 
in the sum on $j$ in which all the spins lie within a correlated spin cluster. A quantitative determination of this contribution can be made by approximating $\left\langle G_{i j}^{(2)}\right\rangle^{+-}$as

$$
\left\langle G_{i j}^{(2)}\right\rangle^{+-}=A_{i j}^{+-}(2)+B_{i j}^{+-}(2),
$$

where

$$
A_{i j}^{+-}(2)=\left\langle G_{i j}^{(2)}\right\rangle_{s w}^{+-} \rho_{i j}
$$

and

$$
B_{i j}^{+-}(2)=\left\langle G_{i j}^{(2)}\right\rangle^{+-}\left(1-p_{i j}\right)
$$

Spin wave theory is used in evaluating $A_{i j}^{+-}(2)$ and the result is

$$
\begin{aligned}
A_{i j}^{+-}(2) & =4 s^{2} \sum_{m n} J_{i n} J_{j m}\left\{( 2 s ) \left[\left\langle a_{n} a_{m}^{\dagger}\right\rangle\right.\right. \\
& \left.\left.-\left\langle a_{i} a_{m}^{\dagger}\right\rangle-\left\langle a_{n} a_{j}^{\dagger}\right\rangle+\left\langle a_{i} a_{j}^{\dagger}\right\rangle\right]\right\}_{i},
\end{aligned}
$$

where as long as $r \ll \lambda, p_{i j}$ is the probability that the spins on sites $1, j, n ; m$ all lle within a currelated spin cluster.

Again since the spins in $8_{i j}^{+-}(2)$ lie outside the spin clusters, Reiter's decoupling scheme can be used to approximate the four-spin correlation functions in terms of products of equilibrium spin pair correlation functions:

$$
\left\langle s_{i}^{+} s_{m}^{z} s_{j}^{-}\right\rangle \simeq\left\langle s_{i}^{+} s_{j}^{-}\right\rangle\left\langle s_{m}^{z} s_{n}^{z}\right\rangle
$$


which implies that the spin pair at sites $i$ and $j$ is statistically independent of the spin pair at $m$ and $n$. Thus

$$
\begin{aligned}
B_{i j}^{+-}(2) & =4 \sum_{m n} J_{i n} J_{j m}\left[\left\langle s_{n}^{+} s_{m}^{-}\right\rangle\left\langle s_{i}^{z} s_{j}^{z}\right\rangle\right. \\
& -\left\langle s_{i}^{+} s_{m}^{-}\right\rangle\left\langle s_{n}^{z} s_{j}^{z}\right\rangle-\left\langle s_{n}^{+} s_{j}^{-}\right\rangle\left\langle s_{i}^{z} s_{m}^{z}\right\rangle \\
& \left.+\left\langle s_{i}^{+} s_{j}^{-}\right\rangle\left\langle s_{n}^{z} s_{m}^{z}\right\rangle\right]\left(1-\dot{\rho}_{i j}\right)
\end{aligned}
$$

Upon taking the Fourier transform of $A_{i j}^{+-}(2)$ and $B_{i j}^{+-}(2)$ we obtain,

$$
\begin{aligned}
& \left\langle\overrightarrow{G^{(2)}}\right\rangle^{+-}=\frac{2 S}{N} \sum_{\vec{q}^{\prime}}\left[\omega\left(\vec{q}^{\prime}\right)\right]^{2}\left[\left\langle\vec{q}_{\vec{q}^{\prime}}\right\rangle+1\right] \rho\left(\vec{q}-\vec{q}^{\prime}\right) \\
& +\frac{8}{N}\left[\frac{1}{3} s(s+1)\right]^{2}\left[\underset{\vec{q}^{\prime}}{\sum}\left[\left(\vec{q}^{\prime}\right)-J\left(\vec{q}-\vec{q}^{\prime}\right)\right]^{2} \rho\left(\vec{q}^{\prime}\right) \rho\left(\vec{q}+\vec{q}^{\prime}\right)\right. \\
& \left.-\sum_{\vec{q}^{\prime} \vec{q}^{\prime \prime}}\left[J\left(\vec{q}^{\prime}\right)-J\left(\vec{q}^{\prime}+\vec{q}^{\prime \prime}\right)\right]^{2} p\left(\vec{q}^{\prime}\right) p\left(\vec{q}^{\prime}+\vec{q}^{\prime \prime}\right) p\left(\vec{q}-\vec{q}^{\prime \prime}\right)\right\} .
\end{aligned}
$$

The first term in the above equation is the fourier transform of $A_{i j}^{+-}(2)$, and thus it gives the spin cluster contribution to $\left\langle\underset{\vec{q}}{\left\langle G^{(2)}\right.}\right\rangle^{+-}$, i.e. it is due to the local order above the critical point. Both the second and third terms on the right hand side of Eq. (IV.58) come from $B_{i j}^{+-}(2)$. It should be noted that the second term is quite similar to the expression Reiter ${ }^{39}$ has derived for the second moment of the spectral density except for the factor $\rho(\vec{q})$ which appears in the denominator of his equation. The third term plays a very important role at temperatures near $T_{c}$ because as $T \rightarrow T_{c}, p(\vec{q})$ becomes strongly peaked at $\vec{q}=0$ for 
a ferromagnet. The width of this peak is measured by $\lambda^{-1}$. Consequently since we are considering only those wave vectors which satisfy $q>\lambda^{-1}$ the third term tends to cancel the second. This behavior will be discussed in detail through the use of a specific model in Appendix B. Therefore, when $T \geqslant T_{c}$ the short range order contribution dominates near the critical temperature, and

$$
\left.\underset{\vec{q}}{\left\langle G^{(2)}\right.}\right\rangle^{+-} \cong \frac{2 S}{N} \underset{\vec{q}^{\prime}}{\sum}\left[\omega\left(\vec{q}^{\prime}\right)\right]^{2}\left[\left\langle n_{\vec{q}^{\prime}}\right\rangle+1\right] \rho\left(\vec{q}-\vec{q}^{\prime}\right) .
$$

In like manner it can be readily shown (Appendix A) that the dominant contribution to $\left\langle\vec{G}{ }_{\vec{q}}^{(0)}\right\rangle^{+-}$is also due to correlated spin clusters:

$$
\left\langle G_{\vec{q}}^{(0)}\right\rangle^{+-}=\frac{2 S}{N} \sum_{\vec{q}^{\prime}}\left[\left\langle n_{\vec{q}^{\prime}}\right\rangle+1\right] \rho\left(\vec{q}-\vec{q}^{\prime}\right)
$$

Now by examining the results we obtained for the first three coefficients, Eqs. (IV.60), (IV.48) and (IV.59), in the series expansion of $\mathrm{g}^{+-}(\overrightarrow{\mathrm{q}}, \mathrm{t})$ we observe that they have a very simple form which leads us to postulate that when $T \gtrsim T_{c}$ and $r<q^{-1}<\lambda$,

$$
\left.\underset{\vec{q}}{\left\langle G^{(m)}\right.}\right\rangle^{+-} \simeq \frac{2 S}{N} \sum_{\vec{q}^{\prime}}\left[w\left(\vec{q}^{\prime}\right)\right]^{m}\left[\left\langle n_{\vec{q}^{\prime}}\right\rangle+1\right]_{p}\left(\vec{q}-\vec{q}^{\prime}\right)
$$

for $n>2$. However, we are not able to give a general proof for this expression because as the order of the coefficient increases more and more spins are contained within a cluster of size $\lambda$, and therefore the cricerion, Eq. (IV.II), which determines the validity of our decoupling procedure will break down eventually, but the discrepancies that do 
:cur should come in the higher order coefficients which affect only the behavior of the wings of the line shape. Consequently, a good result for the central line shape may still be expected. When Eq. (IV.61) is substituted into Eq. (IV.20), then

$$
\begin{gathered}
G^{+-}(\vec{q}, \omega)=\frac{2 S}{N} \sum_{\vec{q}^{\prime}}\left\{\frac{1}{2} \int_{-\infty}^{\infty} d t e^{\left.-i \omega t\left[\sum \frac{(i t)^{m}}{m !}\left(\omega\left(\vec{q}^{\prime}\right)\right)^{m}\right]\right\}}\right. \\
\times\left[\left\langle n_{\vec{q}^{\prime}}\right\rangle+1\right] \rho\left(\vec{q}-\vec{q}^{\prime}\right) .
\end{gathered}
$$

Upon completing the integration we obtain

$$
G^{+-}(\vec{q}, w)=\frac{2 S}{N} \sum_{\vec{q}^{\prime}} \delta\left[w-w\left(\vec{q}^{\prime}\right)\right]\left\{\left\langle n_{\vec{q}^{\prime}}\right\rangle+1\right\} \rho\left(\vec{q}-\vec{q}^{\prime}\right) .
$$

In a similar fashion we can determine $G^{-+}(\vec{q}, \omega)$. The only difference is that the factor $\left\langle n_{\vec{q}}^{\prime}\right\rangle+1$ in Eq. (IV.63) is replaced by $\left\langle n_{\vec{q}}\right\rangle$, and $s\left(w-w\left(\vec{q}^{\prime}\right)\right) \rightarrow \Delta\left(w+\vec{q}^{\prime}\left(\vec{q}^{\prime}\right)\right)$. Thus from Eq. (iv.17, $G^{\top}(\vec{q}, w)$, the time fourier transform of the transverse dynamical spin correlation function, becomes

$$
\begin{aligned}
G^{\top}(\vec{q}, \omega) & =\frac{S}{N} \sum_{\vec{q} l^{\prime}}\left[\left\langle n_{\vec{q}^{\prime}}\right\rangle \delta\left(w-w\left(\vec{q}^{\prime}\right)\right)\right. \\
& \left.+\left\langle n_{\vec{q} !}\right\rangle \delta\left(w+\omega\left(\vec{q}^{\prime}\right)\right)\right\} p\left(\vec{q}-\vec{q}^{\prime}\right) .
\end{aligned}
$$

It should be noted that the above expression contains only the onemagnon contribution to the transverse correlation function. in order to determine the first order nonlinear contribution to $G^{\top}(\vec{q}, w)$, we must include the next order term in the Holstein-Primakoff spin operator 
expansions for $s_{i}^{+}$and $s_{i}^{-}$, so that

$$
\begin{aligned}
& s_{i}^{+} \cong \sqrt{ } \frac{2 S}{N} \sum_{\vec{k}} e^{i \vec{k} \cdot \vec{R}} i \vec{k} \\
& -\frac{1}{N / 8 N S} \vec{k}_{\vec{k}, \vec{k}^{\prime}, \vec{k}^{\prime \prime}} e^{-i\left(\vec{k}-\vec{k}^{\prime}-\vec{k}^{\prime \prime}\right) \cdot \vec{R}_{i}} \quad \vec{k}_{\vec{k}}^{a} \vec{k}^{\prime}{ }^{a} \vec{k}^{\prime \prime},
\end{aligned}
$$

and

$$
\begin{aligned}
& s_{i}^{-} \cong \sqrt{ } \frac{2 S}{N} \sum_{\vec{k}} e^{-i \vec{k} \cdot \vec{R}_{i}} a_{\vec{k}}^{+} \\
& -\frac{i}{N \sqrt{N S S}} \sum_{\vec{k}, \vec{k}^{\prime}, \vec{k}^{\prime \prime}} e^{-i\left(\vec{k}+\vec{k}^{\prime}-\vec{k}^{\prime \prime}\right) \cdot \vec{R}_{i}} \underset{\vec{k}}{a_{\overrightarrow{k^{\prime}}}^{+}{ }^{\dagger} \vec{k}^{\prime \prime}}
\end{aligned}
$$

Then using the same procedure as outlined above we can obtain not only the one magnon contribution but also the lowest order nonlinear contribution to $G^{\top}(\vec{q}, w)$ :

$$
\begin{aligned}
G^{\top}(\vec{q}, \omega) & =\frac{1}{N}\left(S-\frac{1}{N} \sum_{\vec{q}^{\prime \prime}}\left\langle n_{\vec{q}^{\prime \prime}}\right\rangle\right) \sum_{\vec{q}^{\prime}}\left\{\left(\left\langle n_{\vec{q}^{\prime}}\right\rangle+1\right) \delta\left[\omega-\omega\left(\vec{q}^{\prime}\right)\right]\right. \\
& \left.+\left\langle n_{\vec{q}^{\prime}}\right\rangle \delta\left[\omega+\omega\left(\vec{q}^{\prime}\right)\right]\right\} \rho\left(\vec{q}-\vec{q}^{\prime}\right) .
\end{aligned}
$$

The characteristics of the line shape generated by $G^{\top}(\vec{q}, \omega)$ will be discussed in Section $D$. 
C. Determination of $G^{2 z}(\vec{q}, w)$

As in the above discussion for $G^{\top}(\vec{q}, \omega)$, an approximate expression for $\vec{G}^{z z}(\vec{q}, \omega)$ will be obtained by another Taylor series expansion in powers of $t$. The lowest order coefficients in this series will be calculated and used to infer an approximate series expression for the longitudinal spin correlation function. Upon Fourier transformation of this result $G^{z Z}(\vec{q}, w)$ is obtained.

The longitudinal spin correlation function is given by

$$
g^{z z}(\vec{q}, t)=\left\langle s_{\vec{q}}^{z}(0) s_{-q}^{z}(t)\right\rangle
$$

whose Taylor series in powers of $t$ is

$$
g^{z z}(\vec{q}, t)=\sum_{n} \frac{(-i t)^{n}}{n !}\langle\underset{\vec{q}}{(n)}\rangle^{z z}
$$

where

$$
\underset{\vec{q}}{\langle G(n)}\rangle^{z z}=\left[\left(\frac{1}{i} \frac{\partial}{\partial t}\right)^{n}\left\langle\underset{\vec{q}}{\left\langle s^{z}(0)\right.} s_{-\vec{q}}^{z}(t)\right\rangle\right]_{t=0}
$$

The first three coefficients in this series are

$$
\begin{aligned}
& \left\langle G_{\vec{q}}^{(0)}\right\rangle^{z z}=\left\langle s_{\vec{q}}^{z} s^{z} \vec{q}^{2}\right\rangle, \\
& \langle\vec{q}(1)\rangle^{z z}=\left\langle s_{\vec{q}}^{z}\left[H, s_{-\vec{q}}^{z}\right]\right\rangle,
\end{aligned}
$$

nd 


$$
\underset{\vec{q}}{\left\langle G^{(2)}\right\rangle^{z Z}}=\left\langle\underset{\vec{q}}{\left[s^{z}, H\right]}\left[H, s_{-\vec{q}}^{z}\right]\right\rangle .
$$

The calculation of these coefficients closely parallels the calculation carried out for $g^{\top}(\vec{q}, t)$. Consequently, we will only discuss the most salient parts. For example, the spatial fourier transform of $\left\langle G_{\vec{q}}^{(1)}\right\rangle^{z z}$ is given by.

$$
\left.\underset{\vec{q}}{\left\langle G^{(1)}\right.}\right\rangle^{z z}=\sum_{j}\left(G_{i j}^{(i)}\right)^{z z} e^{-i \vec{q} \cdot \vec{R}} i j
$$

where

$$
\begin{aligned}
\left(G_{i j}^{(1)}\right)^{z Z} & =\left\langle s_{i}^{z}\left[H, s_{j}^{z}\right]\right\rangle \\
& =-\sum_{m} J_{j m}\left(\left\langle s_{i}^{z} s_{j}^{+} s_{m}^{-}\right\rangle-\left\langle s_{i}^{z} s_{m}^{+} s_{j}^{-}\right\rangle\right)
\end{aligned}
$$

which has been derived through the use of Eqs. $($ IV ,26) - (IV.28)

From our discussion in Sections $A$ and $B$, the dominant contribution to $\langle\underset{\vec{q}}{(1)}\rangle^{z z}$ comes from clusters of strongly correlated spins. We can thus make the same approximations in order to determine the three-spin static correlation functions. The result we obtain is. 


$$
\begin{aligned}
\left\langle G_{\vec{q}}^{(1)}\right\rangle^{z Z}= & \frac{1}{2 N^{2}} \vec{q}_{\vec{q}^{\prime} \vec{q}^{\prime \prime}}\left\{\left[\omega\left(\vec{q}^{\prime \prime}\right)-\omega\left(\vec{q}^{\prime}\right)\right]\left\langle n_{\vec{q}^{\prime}}\right\rangle\left(\left\langle n_{\vec{q}^{\prime \prime}}\right\rangle+1\right)\right. \\
& \left.+\left[\omega\left(\vec{q}^{\prime}\right)-\omega\left(\vec{q}^{\prime \prime}\right)\right]\left\langle n_{\vec{q}^{\prime \prime}}\right\rangle\left(\left\langle n_{\vec{q}^{\prime}}\right\rangle+1\right)\right\} \\
& \times \rho\left(\vec{q}-\vec{q}^{\prime}+\vec{q}^{\prime \prime}\right) .
\end{aligned}
$$

In a similar manner the dominant contribution to $\left\langle G_{\vec{q}}(0)\right\rangle^{z z}$ and $\langle G \vec{q}(2)\rangle^{z z}$ can be found. The results for these coefficients are obtained in Appendix $C$. Then by taking

$$
\begin{aligned}
\langle\underset{\vec{q}}{(m)}\rangle^{z z} & =\frac{1}{2 M^{2}}{\overrightarrow{q^{\prime}}}_{\vec{q}^{\prime \prime}}\left[\left[w\left(\vec{q}^{\prime}\right)-\omega\left(\vec{q}^{\prime \prime}\right)\right]^{m}\left(1+\left\langle n_{\vec{q}^{\prime}}\right\rangle\right)\left\langle n_{\vec{q}^{\prime !}}\right\rangle\right. \\
& \left.+\left[\omega\left(\vec{q}^{\prime \prime}\right)-\omega\left(\vec{q}^{\prime}\right)\right]^{m}\left(1+\left\langle\vec{n}_{\vec{q}^{\prime \prime}}\right\rangle\right)\left\langle n_{\vec{q} !}\right\rangle\right] \rho\left(\vec{q}+\vec{q}^{\prime}-\vec{q}^{\prime \prime}\right)
\end{aligned}
$$

to be the general form for the coefficients of the powers of $t$ in the series expansion for the longitudinal dynamical spin correlation function, we finally obtain, upon Fourier transformation, the following approximate expression for $G^{2 Z}(\vec{q}, \omega)$ : 


$$
\begin{aligned}
& G^{z Z}(\vec{q}, w)=\left[s-\frac{1}{N} \sum_{\vec{q}^{\prime}}\left\langle n_{\vec{q}^{\prime}}\right\rangle\right]^{2} p(\vec{q}) \delta(w) \\
& +\frac{1}{2 N^{2}} \sum_{\vec{q}^{\prime} \vec{q}^{\prime \prime}}\left\{\delta\left(w+\omega\left(\vec{q}^{\prime \prime}\right)-w\left(\vec{q}^{\prime}\right)\right)\left(1+\left\langle n_{\vec{q}^{\prime}}\right\rangle\right)\left\langle n_{\vec{q}^{\prime \prime}}\right\rangle\right. \\
& \left.+\delta\left(\omega+\omega\left(\vec{q}^{\prime}\right)-\omega\left(\vec{q}^{\prime \prime}\right)\right)\left(1+\left\langle\vec{n}_{\vec{q}^{\prime \prime}}\right\rangle\right)\left\langle n_{\vec{q}^{\prime}}\right\rangle\right\} \\
& x \rho\left(\vec{q}-\vec{q}^{\prime}+\vec{q}^{\prime \prime}\right) \text {. }
\end{aligned}
$$

where the first term in the above expression is due to the HolsteinPrimakoff spin operator expansion for $\mathrm{s}_{\vec{q}}$. The physical implications of this result will be discussed in the next section.

\section{Transverse and. Longitudinal Contributions \\ to the Line Shape}

Using the equations that have been derived for $G^{\top}(\vec{q}, w)$ and $G^{z z}(\vec{q}, w)$ we will determine the frequency distribution and wave vector distribution of the neutron magnetic scattering cross section.

The relevant function which contains all the information concerning these distributions is $G(\vec{q}, w)$ which, as can be seen from Eq. (IV.16) is equal to the sum of $G^{\top}(\vec{q}, \omega)$ and $G^{z Z}(\vec{q}, \omega)$ in our model. Thus in order to gain a quantitative understanding of the line shape characteristics and their physical significance we will consider these functions separately.

In Eq. (IV.67) for $G^{\top}(\vec{q}, w)$, we first vary $w$ keeping $\vec{q}$ fixed which corresponds to a constant momentum scan. When this is done it is 
evident that two peaks will be present in the line shape. The broadening of these peaks is determined not only by the Bose-Einstein distribution function $\left\langle n_{\vec{q}}\right\rangle$ but also by $\rho\left(\vec{q}-\vec{q}^{\prime}\right)$. The contribution of $\rho\left(\vec{q}-\vec{q}^{\prime}\right)$ to this broadening is particularly interesting because we have chosen $\rho_{i j}$ as our local order parameter and so $\rho\left(\vec{q}-\vec{q}^{\prime}\right)$ is a measure of the spatial fluctuations of the correlated spin clusters. Thus these fluctuations of the local order regions contribute directly to the line broadening. Since the width of $\rho\left(\vec{q}-\vec{q}^{\prime}\right)$ is on the order of $1 / \lambda$, the when $1 / \lambda \ll q$, $\rho$ becomes strongly peaked about $\vec{q}^{\prime}=\vec{q}$. Consequently, distinct peaks will be obtained at $\omega= \pm \omega(\vec{q})$ which correspond to the emission and absorption of magnons. When $\vec{q}$ is varied while $\omega$ is fixed two peaks are found at $|\vec{q}|= \pm\left|\vec{q}_{0}\right|$ (when $1 / \lambda \ll q$ ), where $\omega\left(\vec{q}_{0}\right)=\omega$, which are also broadened by $\left\langle n_{\vec{q}}\right\rangle$ and $\rho\left(\vec{q}-\vec{q}^{\prime}\right)$. The factor multiplying the sums over $\vec{q}^{i}$ in $G^{\top}(\vec{q}, \omega)$,

$$
s-\sum_{\vec{q} ! !}\left\langle n_{\vec{q}^{\prime \prime}}\right\rangle
$$

also has physical significance because from elementary spin wave theory we know that

$$
\sum_{\vec{q}^{\prime \prime}}\left\langle n_{\vec{q}^{\prime \prime}}\right\rangle \propto \Delta M=M_{0}-M(T)
$$

where $M_{0}$ is the magnetization for the completely ordered state which in our model would occur at $T=T_{c}$. Therefore

$$
S-\sum_{\overrightarrow{q^{\prime \prime}}}^{\sum}\left\langle n_{\vec{q}^{\prime \prime}}\right\rangle \propto M(T),
$$


and so the size of $G^{\top}(\vec{q}, \omega)$ is modified by a term which is proportional to the temperature dependent magnetization.

The characteristics of the line shape contribution from $G^{22}(\vec{q}, \omega)$ can be discerned directly from Eq. (IV.78). The first term in this expression is a central peak which is a delta function of the frequenc) dt $w=0$, in our approach. In the limit as $T \rightarrow T_{c^{\prime}} \rho(\vec{q}) \rightarrow \delta(\vec{q})$ and the first term gives the elastic Bragg scattering contribution to the cross section. Consequently, when $T>T_{c}$ it is apparent that our central peak is the remnant of the elastic Bragg peak which is diffused spatially due to the fluctuations in the local order parameter as measured by $\rho(\vec{q})$. The model that we have constructed gives a central peak that has a zero frequency width. This impiies that the decay time of the local order parameter is infinite, 42 which is to be expected because our local order parameter is time independent. It is also evident upon examination of the expression for $G^{2 Z}(\vec{q}, \omega)$ that there are two-magnon terms contained in it which are also broadened by convolution with $\dot{\rho}(\vec{q})$.

\section{E. Comparison with Previous Theories}

Although we have not actually determined the moments of the spectral shape function in Sections $B$ and $C$, we can still compare our calculation with moment expansion theories since $G^{\alpha \alpha}(\vec{q}, \omega)$ is closely related to the shape function; $R^{\infty \alpha}(\vec{q}, \omega)$ : 


$$
R^{\alpha \alpha}(\vec{q}, \omega)=\frac{G^{\alpha \alpha}(\vec{q}, \omega)}{\int_{-\infty}^{\infty} G^{\alpha \alpha}(\vec{q}, \omega) d \omega}=\frac{G^{\alpha \alpha}(\vec{q}, \omega)}{\left\langle s_{\vec{q}}^{\alpha} s^{\alpha} \vec{q}^{\prime}\right\rangle} .
$$

Thus we can relate the lowest order coefficients of the powers of $t$ in the Taylor series expansion for the dynamical spin correlation function to the moments of $R^{\alpha \alpha}(\vec{q}, w)$. From Eqs. $(111.36)$ and $(111.40)$ $(111.42)$, we find that the second moment is given by

$$
\left\langle\omega_{\vec{q}}^{2}\right\rangle^{\alpha x x}=\frac{\left\langle G^{(2)}\right\rangle^{\infty \alpha \alpha}}{\left\langle G_{\vec{q}}^{(0)}\right\rangle^{\alpha \alpha x}}
$$

and that the fourth moment is simply

$$
\begin{aligned}
&\left\langle\omega_{\vec{q}}^{4}\right\rangle^{\alpha \alpha}=\frac{\left.\vec{q}{ }^{(4)}\right\rangle^{\alpha \alpha}}{\left\langle G^{(0)}\right\rangle^{(\alpha \alpha}} . \\
& \therefore \quad \vec{q}
\end{aligned}
$$

In Reiter!s derivation of the second and fourth moments of the spectral density above the critical temperature, 40 the fluctuation of the second moment, defined by,

$$
\left\langle\Omega_{\vec{q}}^{4}\right\rangle^{\alpha \alpha}=\underset{\vec{q}}{\left\langle w^{4} \alpha \alpha\right.}-\left(\left\langle w_{\vec{q}}^{2}\right\rangle^{\alpha \alpha}\right)^{2}
$$

satisfies the following relation 



\section{5}

section of $\mathrm{Ni}^{17}$ and $\mathrm{RbMnF}_{3}{ }^{18}$ The most important reason for this difficulty in Reiter's approach can be traced to the method he used to decouple the multiple-spin correlation functions.

As was stated in Section B, Reiter decoupled the four-spin correlation functions in the following way: :

$$
\left\langle s_{n}^{+} s_{l}^{z} s_{m}^{-} s_{j}^{z}\right\rangle \simeq\left\langle s_{n}^{+} s_{m}^{-}\right\rangle\left\langle s_{l}^{z} s_{j}^{z}\right\rangle
$$

of spins. This particular decoupling scheme, that all spin clusters are statistically independent from every other pair, is valid at high temperatures, where there is much spin disorder. In this temperature region the spin correlation length $\lambda$, which is a measure of the size of a correlated spin cluster, is quite small. Therefore, when $q \ll \lambda^{-1}$ where $q$ is the spin fluctuation wave vector, these fluctuations span many spin clusters oriented randomly and thus they must behave quite differently from spin waves.

Reiter's decoupling scheme is quite analogous to one which was developed in order to treat phonon-phonon interactions and which has been quite successful in describing the phenomenon of acoustic attenvation. In studying the attenuation of long wavelength sound waves in. Helium 11 , Kawasaki ${ }^{46-48}$ found that when $q \ll \lambda^{-1}$, where $q$ is the wave vector of the sound wave, and $\lambda^{-1}$ is the inverse correlation length of the particle fluctuations, the correlation functions containing multiple phonon creation and annihllation operators found in the expres-. sion for the attenuation constant can be approximated by products of pair correlation functions. 
76

Although this particular decoupling procedure was found to be quite useful in the acoustic attenuation problem, its application to the problem of spin fluctuations leads to difficulties because it does not adequately account for the positional spin correlations that are impertank when $T \gtrsim T_{c}$ and $q^{-1} \ll \lambda$ which as we have seen must be taken into consideration in the determination of the dynamical spin correlation function. 


\section{DISCUSSION OF RESULTS}

$\therefore$ We now proceed with the evaluation of the expressions we obtained for the Fourier transforms of the transverse and longitudinal dynamical spin correlation functions. In order to accomplish this the sums over the wave vectors $\vec{q}^{\prime}$ and $\vec{q}^{\prime \prime}$ in Eqs. (IV.67) and (IV.78) must be carried out. Since these wave vectors can take on a continuous set of values the sums can be converted to integrals which can be simplified through the use of the following approximations: the spin wave dispersion curve is approximated by a parabola so that

$$
J(0)-J(\vec{q})=\alpha q^{2}
$$

and

$$
\omega_{q}=2 S \alpha q^{2} \equiv D q^{2}
$$

where $\alpha$ and $D$ are fitting parameters and $D$ is often referred to as the spin wave stiffness.

As in other theories dealing with spin-spin interactions above $T_{c}$, our theory requires that we have an explicit expression for the static spin pair correlation function. Consequently we have used a Gaussian function of the form $p(\vec{q}) \propto e^{-\lambda^{2} q^{2}}$ where $\lambda=\left(2 D / k_{B}\left(T-T_{c}\right)\right)^{\frac{1}{2}}$.

These approximations allow us to evaluate the integral expression for $G^{\top}(\vec{q}, \omega)$ analytically, however $G^{2 z}(\vec{q}, \omega)$ must be evaluated through the use of numerical integration techniques. Before we can determine 
the numerical values for these functions, the values of $\lambda$, the two-spin correlation length, and $D$, the spin wave stiffness, must be obtained from experimental data. The spin wave stiffness constant we use is $D=$ $280 \mathrm{meV}-\AA^{2}$. In the case of $\mathrm{Ni}$, neutron inelastic scattering experiments have indicated that at $T_{c}+50 \mathrm{~K}\left(T_{c} \cong 631 \mathrm{~K}\right), \lambda^{-1}=0.11 \mathrm{~A}^{-1}$. The value at any other temperature can then be calculated with the help of the temperature scaling law for the inverse correlation length, Eq. (111.44). (As an interesting aside it should be noted that this temperature dependence yields $\lambda^{-1}=0.15 \AA^{-1}$ at $711 \mathrm{~K}\left(T_{c}+80 \mathrm{~K}\right)$. This result indicates that the high temperature spin wave dispersion curve reported by Mook, Lynn, and Nicklow 17 lies in the short wavelength region $q>\lambda^{-1}$ ). The function $G(\vec{q}, \omega)$ can now be evaluated numerically. In Figure 9 a typical set of line shapes is shown for the case where $\omega$ is held constant and $q$ is varied. (A constant energy scan.) The most salient character- istic of these line shapes is that they contain distinct peaks which are located at $q= \pm q_{0}$, where $\hbar_{\omega}\left(q_{0}\right) \stackrel{d}{=} t_{\omega}$ is the energy of a spin wave whose wave vector is $q_{0}$.

Although these line shapes have the same qualitative appearance as those obtained experimentally in the inelastic scattering of neutrons from $\mathrm{Ni}$, a more detailed comparison reveals definite discrepancies between our simple theory and the $\mathrm{Ni}$ data. In Figure 10 we compare our numerical results for the full-width-at-half-maximum (FWHM) for the peak in $G(\vec{q}, w)$ at various. temperatures with the experimental curve for $N i$ for an energy transfer $\Delta E=\hbar_{\omega}=12.4 \mathrm{meV}$, where the instrumental resolution 


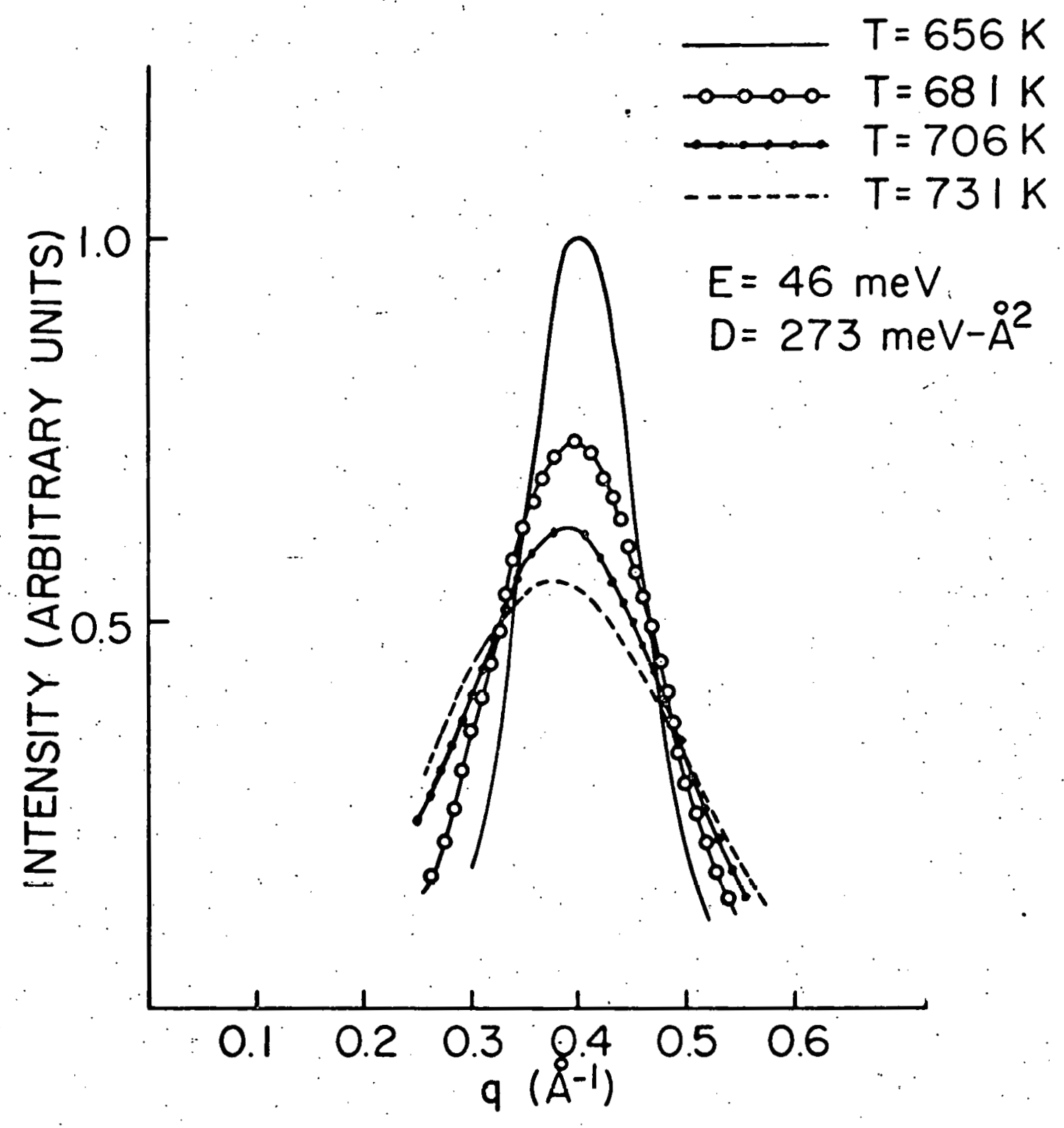

Figure 9. Temperature dependence of the theoretical line shape. 


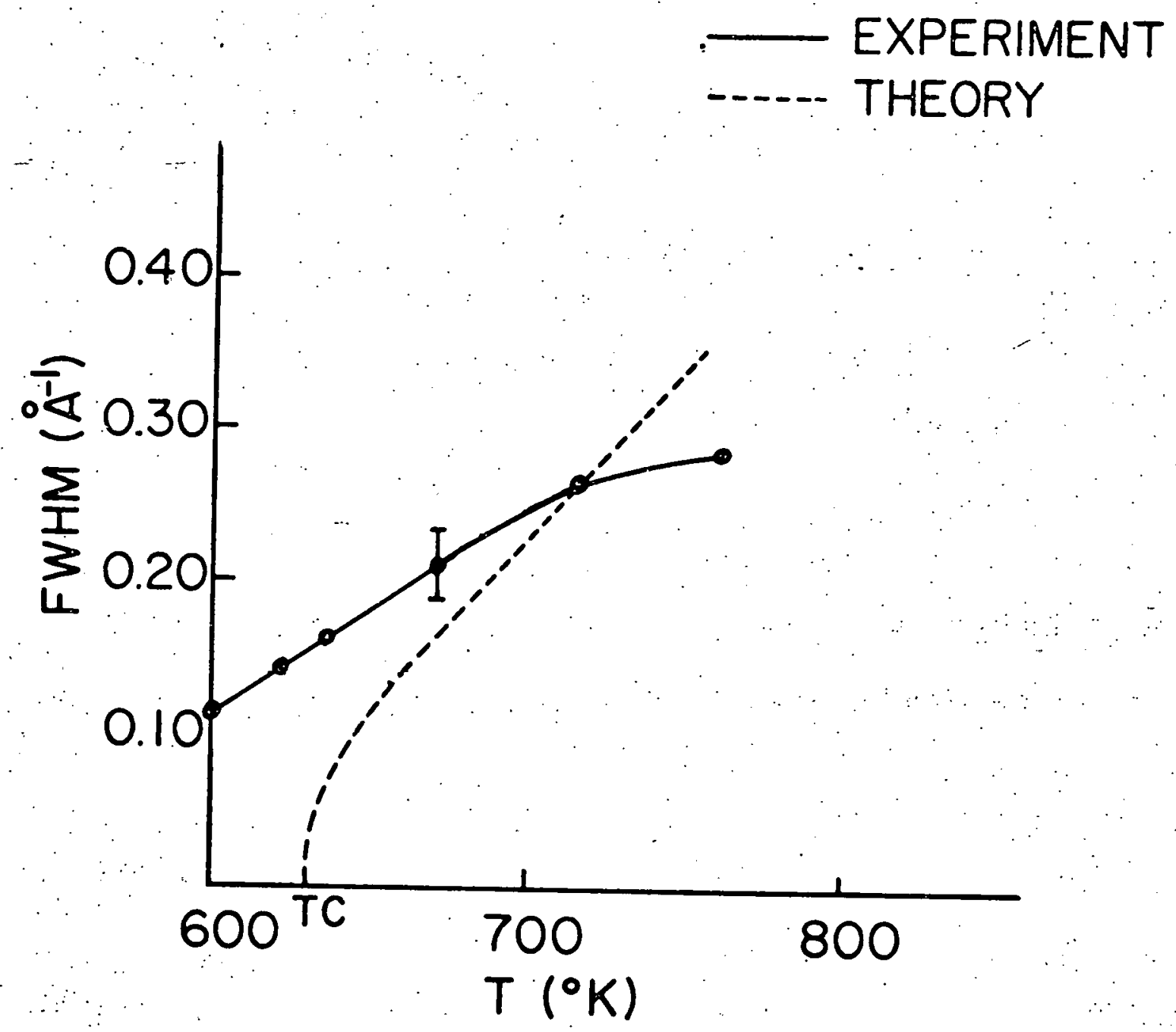

Figure 10. Comparison between the experimental and theoretical line widths in $\vec{q}$ as a function of temperature for nickel at an energy transfer of $\Delta E=12.4 \mathrm{meV}$. 
tos been subtracted out. In the region from $656^{\circ} \mathrm{K}\left(1.03 \mathrm{~T}_{\mathrm{c}}\right)$ to $706^{\circ} \mathrm{K}$ $\left(1.12 T_{c}\right)$ the slope of the curve produced by our theory compares favorably with the experimental curve. However, below these temperatures the line width of the theoretical peak approaches zero as $T \rightarrow T_{c}$. This is not surprising because in applying spin wave theory to evaluate the spin correlation functions involving spins within a correlated cluster, we have included only the lowest order linear and nonlinear terms in the Wolstein-Primakoff spin operator expansions. By doing this we have constructed a theory in which the spins within a cluster are strongly correlated and thus they tend to be aligned in one particular direction defined to be the local $z$ axis of the cluster. Consequently, at $T=T_{c}$ the spin alignment in our model corresponds to that which actually exists near $T=0^{\circ} \mathrm{K}$. This observation implies that our theory should work better in the case of one dimensional magnetic systems where $T_{c}=0^{\circ} \mathrm{K}$.

The other deviation from the experimental results is observed above $706^{\circ} \mathrm{K}$. In this high temperature region the theoretical curve has the same slope as it did when $T<706^{\circ} \mathrm{K}$ because the linewidths of the theoretical peaks continue to increase at the same rate. However, the experimental curve bends over, because the experimental linewidths are observed to remain almost constant up to the highest temperature used $\left(2 \mathrm{~T}_{c}\right)$. A possible reason for this discrepancy lies in our choice of a static spin correlation function for the measurement of the probability that the spins within a spin cluster are correlated. Consequently, in 
82

our simple theory, line broadening is only affected by one parameter, the spin correlation length $\lambda$. As the temperature increases, $\lambda$ decreases, and this tends to broaden the line because the clusters become smaller. The peaks will continue to broaden as the temperature increases. However, in a more refined calculation a time dependent spin correlation function must be used to specify the probability because above $T_{c}$ spin diffusion causes the spin clusters to decay with time. Thus as the temperature increases the tendency for the line to broaden due to a decrease in the correlation length is balanced by the increase in spin diffusion which effectively allows only the large clusters to sustain a spin wave excitation while in the small clusters this cannot occur. Hence the line should not broaden very much as the temperature increases. 
VI. CONCLUSION

The single most important result obtained from the foregoing calculation is that above the critical temperature when $r<q^{-1}<\lambda$ is satisfied, positional correlations between spins must be considered because these correlations directly effect the shape of the neutron scattering cross section. Within this wave vector region we have found that when the localized spin system is approximated by clusters of correlated spins whose degree of order is measured by a time-independent order parameter, the two spin correlation function $\rho(\vec{q})$, then the fourier transform, $G(\vec{q}, w)$, of the dynamical spin correlation function, obtained through the use of this approximation, exhibited distinct spin wave like peaks. The full-width-at-half-maximum of these peaks and their position compare qualitatively with the experimental results obtained by Mook, Lynn, and Nicklow. 17 A more detailed comparison reveals that we can only achieve a quantitative agreement betwcen experimenl and theory by refining the approximatlons that have been used. These include the following.

1. Magnon-magnon interactions have been neglected. This causes our line-widths to approach zero as $T \rightarrow T_{C^{*}}$

2. The higher order coefficients in the time Fourier series expansion of the dynamical spin correlation function involve progressively larger numbers of spins within the cluster. Hence our approximation that $r<\lambda$ must breakdown at some order. 
3. The temperature within a cluster has been assumed to be the same as that of the whole crystal. This approximation is valid as long as , there are no strong temperature fluctuations over small regions in the system and the clusters are large.

4. The local order parameter used in this theory was the static spin-pair correlation function. This approximation is one of the major causes for the continued. increase in the theoretical line-widths as the temperature increases. As we pointed out in Chapter $V$ this aproximation can be refined through the use of a spin diffusion correlation function.

It is also important to note that the theory developed here is applicable to simple Heisenberg ferromagnets. Consequently, it is not surprising that only quantitative agreement with the inelastic neutron scattering data is obtained with $\mathrm{Ni}$ because although nickel does exhibit characteristics of localized spin systems, it is well known that the magnetic moment per atom in $\mathrm{Ni}$ is not an integral number of Bohr magnetons which implies the d-electrons are itinerant. Furthermore, neutron scattering experiments on $\mathrm{Ni}$ have found a sharp cutoff in spin wave intensities above a certain energy which is predicted by the Stoner model of intinerant ferromagnetism in which a sharp decrease in intensity occurs when the spin wave dispersion curve intersects a continuum band of Stoner excitations. Our model in its present form can not predict such. a sharp cutoff. These findings indicate that a complete theory dealing with spin fluctuations in metals must explicityy consider the itinerant nature of the conduction electrons. 
Finally, because of the simplicity of the theory we have developed we should be able to readily modify it so that we can study the magnetic excitations of systems in which the crystal field only ground state is a singlet. In these systems it has been found ${ }^{49-51}$ that the existence of a magnetic phase transition is dependent upon the magnitude of the ratio of the exchange interaction to the crystal field interaction which are usually of comparable size. When the exchange interaction is small this ratio is less than a certain threshold value 51 and no magnetic ordering occurs, however when the ratio exceeds this value a magnetic phase transition is expected where the ordering is due to a polarization (induced moment) instability in the ground state wave function. In the theories ${ }^{52-55}$ that have been proposed this phase transition is driven by the softening of that magnetic exciton mode whose wave vector $\vec{q}$ corresponds to the wave vector of the ordered structure. This soft-mode behavior has, however, not been observed experimentally. $56-58$ it is important to note that in these inelastic neutron scattering experiments the scattering wave vector used was $q>0.5 \AA^{-1}$, and thus only short wavelength magnetic exciton modes were being excited. Consequently it would be quite interesting to examine the effect of positional correlations between the induced moments on the line shape of the neutron scattering cross section through. the use of our theory. 

20. M. T. Hutchings, G. Shirane, R: J. Birgeneau and S. L. Holt, Phys. Rev. B5, 1999 (1972).

21. R. J. Birgeneau, R. Dingle, M. T. Hutchings, G. Shirane and S. L. Holt, Phys. Rev. Letters 26, 718 (1971).

22. See for example, L. D. Landau and E. M. Lifshitz, Statistical Physics (Pergamon, London 1964), p. 482.

23. H. Tomita and. H. Mashiyama, Prog. Theor. Phys. 48, 1133 (1972).

24. W. Marshall, Natn. Bur. Stand. Misc. Publ. No. 273, 135 (1966).

25. P. Resibois and M. De Leener, Phys. Rev. 152, 305 (1966).

26. P. Resibois and M. De Leener, Phys. Rev. 152, 318 (1966)。

27. P. Resibois and M. De Leener, Phys. Rev, 178, 806 (1969).

28. P. Resibois and M. De Leener, Phys. Rev. 178, 819 (1969).

29. M. Blume and J. Hubbard, Phys. Rev. Bl, 3815 (1970).

30. R. Kubo, J. Phys. Soc. Japan 12, 570 (1957).

31. P. G. De Gennes, J. Phys. Chem. Solids 4, 223 (1958).

32. H. S. Bennett, Phys. Rev. 174, 629 (1968).

33. B. 1. Halperin and P. C. Hohenberg, Phys. Rev. 177, 952 (1969).

34. K. Tomita and H. Tomita, Prog. Theor. Phys. 45, 1407 (1971).

35. H. Mori, Prog. Theor. Phys. 33, 423 (1965).

36. M. Dupuis, Prog. Theor. Phys. 37, 520 (1967).

37. S. W. Lovesey and R. A. Meserve, J. Phys. C (Proc. Phys. Soc.) 6 , 79 (1973).

38. M. F. Collins and W. Marshal1, Proc. Phys. Soc. 22,390 (1967).

39. G. F. Reiter, Phys. Rev. B5, 222 (1972).

40. G. F. Reiter, Phys, Rev. B7, 3325 (1973).

41. M. E. Fisher, Am. J. Phys. 32, 343 (1964). 
42. F. B. MeLean and M. Blume, Phys. Rev. B7, 1149 (1973).

43. L. Van Hove, Phys. Rev. 25, 1374 (1954).

44. W. Marshall and R. D. Lowde, Rept. Prog. Phys. 31, 705 (1968).

45. L. P. Kadanoff, W. Götze, D. Hamblen, R. Hecht, E. A. S. Lewis, V. V. Palciauskas, M. Rayl and J. Swift, Rev. Mod. Phys. 32, 395 (1967).

46. K. Kawasaki, Prog. Theor. Phys. (Japan) 26, 793 (1961).

47. K. Kawasaki, Prog. Theor. Phys. (Japan) 26, 795 (1961).

48. K. Kawasaki and H. Mori, Prog. Theor. Phys. (Japan) 28, 784 (1962).

49. G. T. Tramme 1, J. App 1. Phys. 3i, 3625 (1960).

50. B. Bleaney, Proc. Roy. Soc. (London) A276, 19 (1963).

51. 8. R. Cooper, Phys. Rev. 163, 444 (1967). For a recent review see B. R. Cooper and.0. Vogt, J. Phys. (Paris) $32, \mathrm{Cl-958} \mathrm{(1971).}$

52. D. A. Pink, J. Phys. C1, 1246 (1968).

53. Y. -L. Wang and B. R. Cooper, Phys. Rev. 172, 539 (1968).

54. Y. -L. Wang and B. R. Cooper, Phys. Rev. 185, 696 (1969).

55. Y. Y. Hsieh and M. Blum, Phys. Rev. 86; 2684 (1972).

56. R. J. Birgeneau, J. Als- Nielson, and E. Bucher, Phys. Rev. Letters, 27, 1530 (1971).

57. B. R. Cooper, Phys. Rev. B6, 2724 (1972).

58. R. J. Birgeneau, J. Als - Nielsen, E. Bucher, Phys. Rev. B6, 2724 (1972). 


\section{VIII: ACKNOWLEDGMENTS}

The author wishes to thank Dr. S. H. Liu for his suggestion of this problem and for his continued guidance, generous support, and enthusiasm throughout all parts of this work. The author is extremely grateful for the many beneficial and informative conversations that he has had with Dr. Liu.

The author also deeply appreciates the support that he has received from his wife, Elizabeth. Her warm encouragement and vast patience helped the author get through many difficult times during the course of this work. 
1X. APPENDIX A: CALCULATION OF $\underset{\vec{q}}{\left\langle G^{(0)}\right\rangle^{+-}}$AND $\left\langle\underset{\vec{q}}{\left\langle G^{(3)}\right.}\right\rangle^{+-}$

From Eq. $(111.40)$ we know that

$$
\left.\langle\vec{q}(0)\rangle=\underset{\vec{q}}{\left\langle S^{+} s^{-}\right.}\right\rangle
$$

We can approximate this expansion coefficient in the manner discussed in Chapter IV by taking the positional correlations of the spins explicitly into consideration:

$$
\langle\vec{q}(0)\rangle^{+-}=\sum_{j}\left[A_{i j}^{+-}(0)+B_{i j}^{+-}(0)\right] e^{-i \vec{q} \cdot \vec{R}_{i j}},
$$

where

$$
A_{i j}^{+-}(0)=\left\langle S_{i}^{+} s_{j}^{-}\right\rangle_{s w} P_{i j}
$$

and

$$
B_{i j}^{+}(0)=\left\langle s_{i}^{+} s_{j}^{-}\right\rangle\left(1-\rho_{i j}\right)
$$

in which $\rho_{i j}$ is defined by Eq. (IV.46) in Chapter IV. The correlation function $\left\langle S_{i}^{+} s_{j}^{-}\right\rangle$in $A_{i j}^{+-}(0)$ can be evaluated through the use of spin wave theory since the spins at sites $i$ and $j$ lie within a correlated cluster. In $B_{i j}^{+\infty}(0)$ the spin conrelation function is evaluated in terms of an equilibrium spin pair correlation function, Eq. (IV.47). When the Holstein-Primakoff spin operator expansion, Eqs. (IV.65) and (IV.66) are employed we obtain 


$$
\begin{aligned}
& A_{i j}^{+-}(0)=\left\{\frac{2 S}{N} \sum_{\vec{k}, \vec{k}_{1}} e^{i \vec{k} \cdot \vec{R}_{i}} e^{-i \vec{k}_{1} \cdot \vec{R}_{j}}\left\langle\underset{\vec{k}}{a_{\vec{k}}} \vec{a}_{1}^{+}\right\rangle\right. \\
& =\frac{1}{2 N^{2}} \sum_{\vec{k}, \vec{k}_{1}} e^{i \vec{k} \cdot \vec{R}_{i}} e^{-i\left(\vec{k}_{1}+\vec{k}_{2}-\vec{k}_{3}\right) \cdot \vec{R}_{j}} \\
& \left\langle\begin{array}{llll}
\vec{k}_{\vec{k}} & \vec{k}_{1}^{+} & \vec{k}_{2}^{+} & \vec{k}_{3}
\end{array}\right. \\
& -\frac{1}{2 N^{2}} \sum_{\vec{k}, \vec{k}_{1}} e^{-i\left(\vec{k}_{1}-\vec{k}_{2}-\vec{k}_{3}\right) \cdot R_{i}} e^{-i \vec{k} \cdot \vec{R}} j \\
& \vec{k}_{2}, \vec{k}_{3}
\end{aligned}
$$

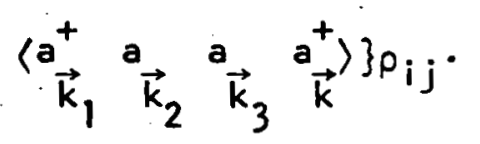

Thus when the sum in $j$ in Eq. (A.1) is carried out the following expression for $\langle\underset{\vec{q}}{(0)}\rangle^{+-}$results:

$$
\begin{aligned}
& \left.\underset{\vec{q}}{\left\langle G^{(0)}\right.}\right\rangle^{+-}=\frac{2}{N}\left(S-\frac{1}{N} \underset{\vec{q}}{\sum_{\vec{q}}}\left\langle n_{\vec{q}}^{\prime \prime}\right\rangle\right) \underset{\vec{q}^{\prime}}{\sum}\left(\left\langle n_{\vec{q}}^{\prime}\right\rangle+1\right)_{p}\left(\vec{q}-\vec{q}^{\prime}\right) \\
& +\left[\frac{2}{3} S(S+1)\right]\left(_{p}(\vec{q})=\frac{1}{N} \underset{\vec{q}}{\sum} p\left(\vec{q}^{\prime}\right)_{p}\left(\vec{q}-\vec{q}^{\prime}\right)\right)
\end{aligned}
$$

which contains those terms which make a non-zero contribution to the series expansion of the time dependent correlation function. The last term in the above expression, as discussed in Appendix B, approaches zero as $T \rightarrow T_{c}$, so when $T Z_{c}$ and $q>\lambda^{-1}$ the first term dominates. 
The expansion coefficient $\langle\underset{\vec{q}}{\langle(3)}\rangle^{+-}$can also be approximated in the same manner, i.e.

$$
\langle\underset{\vec{q}}{(3)}\rangle^{+-}=\sum_{j}\left[A_{i j}^{+-}(3)+B_{i j}^{+-}(3)\right] e^{-i \vec{q} \cdot \vec{R}_{i j}}
$$

where

$$
A_{i j}^{+-}(3)=\left\langle\left[S_{i}^{+}, H\right]\left[H,\left[H, S_{j}^{-}\right]\right]\right\rangle_{s w} \rho_{i j}
$$

and

$$
\mathrm{B}^{+-}(3)=0
$$

by symmetry. The reason why $\mathrm{B}_{i j}^{+-}(3)$ is zero is discussed in detail in Chapter IV. Since $H$ is the Heisenberg Hamiltonian, then after some manipulation, spin operator commutator algebra yields

$$
\begin{aligned}
& A_{i j}^{+-}(3)=\left\{8 \sum_{l p r} J_{i l} J_{j p} J_{j r}\left\langle\left(s_{l}^{+} s_{i}^{2}-s_{i}^{+} s_{l}^{2}\right)\left(s_{r}^{-} s_{j}^{2}-s_{j}^{-} s_{r}^{2}\right) s_{p}^{2}\right\rangle,\right. \\
& -4 \underset{l p r}{\Sigma} J_{i l} J_{j p} J_{p r}\left\langle\left(s_{l}^{+} s_{1}^{z}-s_{i}^{+} s_{l}^{z}\right) s_{j}^{-}\left(s_{p}^{+} s_{r}^{-}-s_{r}^{+} s_{p}^{-}\right)\right\rangle \\
& -8 \sum_{\ell p r} J_{i l} J_{p r}\left\langle\left(s_{l}^{+} s_{i}^{z}-s_{i}^{+} s_{l}^{z}\right)\left(s_{r}^{-} s_{p}^{z}-s_{p}^{-} s_{r}^{z}\right) s_{j}^{z}\right\rangle \\
& \left.-4 \underset{l p r}{\sum_{i l}} j_{j p} J_{j r}\left\langle\left(s_{l}^{+} s_{i}^{z}-s_{i}^{+} s_{l}^{z}\right) s_{p}^{-}\left(s_{j}^{+} s_{r}^{-}-s_{r}^{+} s_{j}^{-}\right)\right\rangle\right\} \rho_{i j}
\end{aligned}
$$


Then using the spin operator expansions given by Eqs. (IV.37), (IV.65), (IV.66) and completing the sums on $j, l, p$, and $r$, we obtain

$$
\begin{gathered}
\left\langle\vec{G}_{\vec{q}}^{(3)}\right\rangle^{+-}=\frac{2}{N}\left(s-\frac{1}{N} \sum_{\overrightarrow{q^{\prime}}}\left\langle n_{\vec{q}}{ }^{\prime \prime}\right\rangle\right) \sum\left(\vec{q}^{\prime}\left(\vec{q}^{\prime}\right)\right)^{3} \\
\times\left(\left\langle\vec{n}_{\vec{q}}^{\prime}\right\rangle+1\right)_{p}\left(\vec{q}-\vec{q}^{\prime}\right)
\end{gathered}
$$

which again contains those terms which make a finite contribution to the Taylor series expansion of the dynamical spin correlation function. it is quite evident that an explicit calculation of the higher order expansion coefficients becomes extremely tedious due to the number of terms contained in the multiple commutator expressions. 
X. APPENDIX B: TEMPERATURE DEPENDENCE OF THE BACKGROUND TERMS IN $\underset{\vec{q}}{(0)}$ ?

$$
\text { AND }\langle\underset{\vec{q}}{(2)}\rangle^{+-}
$$

In the expression Eq. (A.6) we obtained for $\langle\underset{\vec{q}}{(0)}\rangle^{+-}$the term that was neglected when we determined the Taylor series expansion of the dynamical spin correlation function has the form

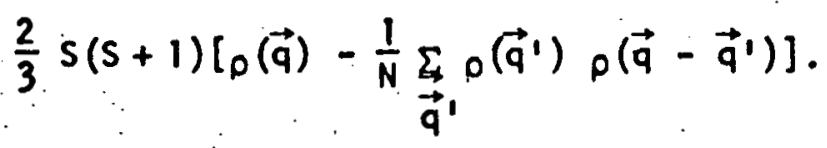

This is the same, except for multipicative constant, as the term that was neglected in $\langle\vec{q}(0)\rangle^{2 z}$. The reason for this comes from the fact that above $T_{c}$, when the spins lie outside the correlated cluster, the correlation between pairs of spins satisfies the following equality:

$$
\left\langle s_{i}^{x} s_{j}^{x}\right\rangle=\left\langle s_{i}^{y} s_{j}^{y}\right\rangle=\left\langle s_{i}^{z} s_{j}^{z}\right\rangle
$$

In order to examine the size of Eq. (B.1) and its temperature dependence a specific model will be used. For simplicity we take $p(\vec{q})$ to have a Gaussian. form so $\rho(\vec{q}) \propto e^{-\lambda^{2} q^{2}}$ where $\lambda$ is the two-spin. correlation length. Then the integral that results when the summation on $\vec{q} \cdot$ is converted to an integration can be solved analytically. The dominant term [see Eq. (A.6)] can also be evaluated using this model, however the value for this term must be determined through the use of numerical integration techniques. When these calculations are carried 
out we find that at $T_{c}+25 \mathrm{~K}(656 \mathrm{~K})$ the ratio of the background term to the term we have retained is on the order of 0.01 . However, the background is very temperature sensitive because at $T_{c}+40 \mathrm{~K}(671 \mathrm{~K})$ this ratio is 0.15 , and as the temperature increases to $T_{c}+100^{\prime} \mathrm{K}$ the background term is no longer negligible. In general as long as $q \lambda \gtrsim 4.0$ and $\lambda^{-1} \leq 0.10$ this term makes only a small contribution which can be neglected.

The temperature dependence of the background term in $\underset{\vec{q}}{\left\langle G^{(2)}\right\rangle^{+-}}$can also be examined through the use of the above method. The integrations that arise in this analysis are again greatly simplified by the use of a Gaussian spin correlation function and the approximation, $J(0)-j(\vec{q})=$ $\alpha q^{2}$. When this is done the value of the background term can be determined via numerical integration, and the ratio of this term to the dominant contribution to $\left\langle\underset{\vec{q}}{\left\langle G^{(2)}\right.}\right\rangle^{+-}$is found to be 0.02 at $T_{c}+25 K$. Thus at temperatures near $T_{c}$, the contribution of the background term is smali and may be neglected, however it should be noted that this term is also very strungly temperature dependent, and as the temperature increases the background contribution becomes significant. At $\mathrm{T}_{\mathrm{c}}+40 \mathrm{~K}$ the background term is on the order of the term we have retained in the expression for $\left\langle G_{\vec{G}}^{(2)}\right\rangle^{+-}$. Consequently, the expressions we have used for the expansion coefficients in the Taylor series expansion of the dynamical spin correlation function are very good approximations only at temperatures near the critical temperature. 


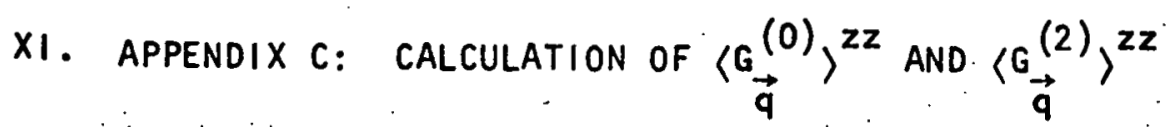

The zeroth order coefficient in the time Taylor series expansion of the longitudinal dynamical spin correlation function is approximated in the usual way:

$$
\underset{\vec{q}}{\langle G(0)})^{z Z} \cong \sum_{j}\left[\left(A_{i j}^{z z}(0)+B_{i j}^{z z}(0)\right)\right] e^{-i \vec{q} \cdot \vec{R}} i j,
$$

where

$$
A_{i j}^{z z}(0)=\left\langle S_{i}^{z} s_{j}^{z}\right\rangle_{s w} p_{i j}
$$

and

$$
B_{i j}^{z z}(0)=\left\langle s_{i}^{z} s_{j}^{z}\right\rangle\left(1-p_{i j}\right)
$$

Spin wave theory is again used to evaluate the spin correlation function in $A_{i j}^{z Z}(0)$ and $B_{i j}^{z Z}(0)$ is evaluated in terms of an equilibrium correlation function, Eqs. (IV.46) and (IV.47). Thus when the Holstein-Primakoff spin operator expansion for $s_{i}^{z}$ is employed we obtain

$$
\begin{aligned}
& A_{i j}^{z z}(0)=\left\{s^{2}-\frac{2 S}{N} \underset{\vec{k}}{\sum}\left\langle n_{\vec{k}}\right\rangle+\frac{1}{N^{2}} \sum_{\vec{k}, \vec{k}^{\prime}}\left\langle n_{\vec{k}}\right\rangle\left\langle n_{\vec{k}^{\prime}}\right\rangle\right. \\
& +\frac{1}{N^{2}} \sum_{\vec{k}, \vec{k}^{\prime}} e^{-i\left(\vec{k}-\vec{k}^{\prime}\right) \cdot R_{i j}}\left(1+\left\langle\vec{n}_{\vec{k}^{\prime}}\right\rangle\right) \\
& x\left\langle\underset{\vec{k}}{\left.\left\langle n_{j}\right\rangle\right\}_{\rho_{j}}}\right.
\end{aligned}
$$


When this is substituted into Eq. (C.1) and the sum on $j$ is carried out we find that

$$
\begin{aligned}
& \left.\underset{\vec{q}}{\left\langle{ }_{G}(0)\right.}\right\rangle^{z Z}=\left[S-\frac{1}{N} \sum_{\vec{q}}\left\langle n_{\vec{q}^{\prime}}\right\rangle\right]^{2} p(\vec{q}) \\
& \left.+\frac{1}{N^{2}} \underset{\vec{q}^{\prime} \vec{q}^{\prime \prime}}{\sum}\left\langle n_{\vec{q}^{\prime}}\right\rangle\left(\ln _{\vec{q}^{\prime \prime}}\right\rangle+\beta\right)_{p}\left(\vec{q}+\vec{q}^{\prime}-\vec{q}^{\prime \prime}\right) \\
& +\left[\frac{2}{3} s(s+1)\right]\left(\rho(\vec{q})-\frac{1}{N} \sum \rho\left(\vec{q}^{\prime}\right) \rho\left(\vec{q}-\vec{q}^{\prime}\right)\right)
\end{aligned}
$$

where the last term in this equation makes only a small contribution when $T \gtrsim T_{c}$ and $q>\lambda^{-1}$. (See Appendix $B_{0}$ )

In like manner we can write

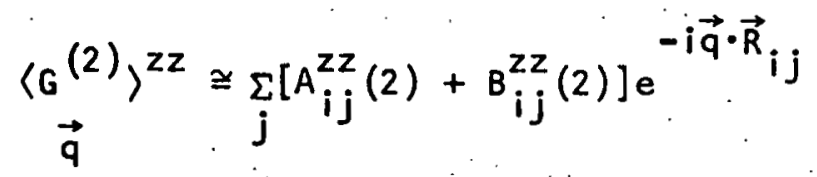

where

$$
A_{i j}^{Z Z}(2)=\left\langle\left[S_{i j}^{Z}, H\right]\left[H, S_{j}^{Z}\right]\right\rangle_{s w} \rho_{i j}
$$

and

$$
B_{i j}^{Z Z}(2)=\left\langle\left[s_{i}^{Z}, H\right]\left[H, s_{j}^{z}\right]\right\rangle\left(1-\rho_{i j}\right) .
$$

Since

$$
\left[s_{i}^{z}, H\right]=\sum_{n} J_{i n}\left(S_{i}^{+} s_{n}^{-}-s_{n}^{+} s_{i}^{-}\right)
$$



where the last two terms tend to cancel each other as $T \rightarrow T_{c}$ and $q>\lambda^{-1}$. 